\title{
LÉVY-BASED COX POINT PROCESSES
}

\author{
GUNNAR HELLMUND, * \\ MICHAELA PROKEŠOVÁ ** AND \\ EVA B. VEDEL JENSEN, ${ }^{* * * *}$ University of Aarhus
}

\begin{abstract}
In this paper we introduce Lévy-driven Cox point processes (LCPs) as Cox point processes with driving intensity function $\Lambda$ defined by a kernel smoothing of a Lévy basis (an independently scattered, infinitely divisible random measure). We also consider log Lévydriven Cox point processes (LLCPs) with $\Lambda$ equal to the exponential of such a kernel smoothing. Special cases are shot noise Cox processes, log Gaussian Cox processes, and log shot noise Cox processes. We study the theoretical properties of Lévy-based Cox processes, including moment properties described by $n$ th-order product densities, mixing properties, specification of inhomogeneity, and spatio-temporal extensions.

Keywords: Cox process; infinitely divisible distribution; inhomogeneity; kernel smoothing; Lévy basis; log Gaussian Cox process; mixing; product density; shot noise Cox process
\end{abstract}

2000 Mathematics Subject Classification: Primary 60D05; 60G55

Secondary $60 \mathrm{~K} 35$

\section{Introduction}

Cox point processes constitute one of the most important and versatile classes of point process models for clustered point patterns [12], [13], [29], [31], [32], [41]. During the last decades, several new classes of Cox point process models have appeared in the literature, e.g. shot noise Cox processes defined by means of generalized gamma measures [5], log Gaussian Cox processes [9], [33], and shot noise Cox processes [28]. These models share some common properties and differ in others, depending on how the driving intensity measure of the Cox process is constructed. One of the aims of this paper is to introduce a unified framework which is able to include all the different models mentioned above, thereby allowing us to show the models in a new light, investigate their relationships, and define further natural extensions.

The starting point for us will be the notion of a Lévy basis $L$, an independently scattered, infinitely divisible random measure. The short terminology of a Lévy basis has been introduced in [2] and [3]. Independently scattered, infinitely divisible random measures have been studied in detail in [36]. Lévy bases include Poisson random measures, mixed Poisson random measures, and Gaussian random measures, as well as the so-called $G$-measures [5]. Thus, having in mind the construction of the shot noise Cox processes, the second step in defining

Received 16 October 2007; revision received 17 April 2008.

* Postal address: T. N. Thiele Centre for Applied Mathematics in Natural Science, Department of Mathematical Sciences, University of Aarhus, Ny Munkegade, Building 1530, DK-8000 Aarhus C, Denmark.

** Current address: Charles University, Faculty of Mathematics and Physics, Sokolovská 83, 18675 Praha 8, Czech Republic.

*** Email address: eva@imf.au.dk 
the driving intensity $\Lambda$ of the Cox process should be a kernel smoothing of the Lévy basis

$$
\Lambda(\xi)=\int k(\xi, \eta) L(\mathrm{~d} \eta),
$$

where $k$ is a kernel (weight) function. By this we arrive at the definition of the Lévy-driven Cox processes (LCPs), i.e. Cox processes with the random driving intensity function defined by an integral of a weight function with respect to a Lévy basis. This construction has been discussed by Wolpert [48] under the name of Lévy moving average processes; see also [49] and [50]. It will be shown that LCPs are, under regularity conditions, shot noise Cox processes with additional random noise.

Furthermore, it is also possible to define the driving intensity as the exponential of a kernel smoothing of a Lévy basis (now allowing for nonpositive weight functions and nonpositive Lévy bases), thus, arriving at the log Lévy-driven Cox processes (LLCPs). It will be shown that LLCPs have, under regularity conditions, a driving field of the form $\Lambda=\Lambda_{1} \Lambda_{2}$, where $\Lambda_{1}$ and $\Lambda_{2}$ are independent, $\Lambda_{1}$ is a $\log$ Gaussian field, and $\Lambda_{2}$ is a $\log$ shot noise field. The latter process may describe clustered point patterns with randomly placed empty holes.

Shot noise Cox processes, log Gaussian Cox processes, and log shot noise Cox processes will appear as natural building blocks in a modeling framework for Cox processes. Different types of combinations of the building blocks (corresponding to thinning and superposition) will be discussed in the present paper.

Having defined the framework, the second aim of this paper is to study the theoretical properties of Lévy-based Cox processes, including moment properties described by $n$ th-order product densities, mixing properties, specification of inhomogeneity, and spatio-temporal extensions.

Examples where the new models are needed already appear in the literature. In [47] an LCP (Cox process with additional random noise) is used in the modeling of a tropical rain forest. In [13, pp. 92-100] a point pattern from forestry is described by a shot noise Cox process thinned by a random field, taking unexplained large-scale environmental heterogeneity into account. If this field is log Gaussian, the resulting process is one of the combinations of LCPs and LLCPs to be described in the present paper. In the very recent review paper [32], a tropical rain forest is modeled by inhomogeneous shot noise Cox processes, obtained by thinning of a homogeneous shot noise Cox process with a log-linear deterministic field depending on explanatory variables. If the deterministic field is substituted with a log Gaussian field, we again arrive at a combination of an LCP and an LLCP.

The present paper is organized as follows. In Section 2 we give a short overview of the theory of Lévy bases and integration with respect to such bases. In Section 3 we recall standard results about Cox processes. In Sections 4 and 5 we introduce and study the Lévy-driven Cox processes and the log Lévy-driven Cox processes, respectively. Combinations of LCPs and LLCPs are discussed in Section 6, while inhomogeneous LCPs and LLCPs are considered in Section 7. We conclude in Section 8 with a discussion.

\section{Lévy bases}

This section provides a brief overview of the general theory of Lévy bases, in particular, the theory of integration with respect to Lévy bases. For a more detailed exposition, see [2], [36], [40], and the references therein.

Let $\mathcal{R}$ be a Borel subset of $\mathbb{R}^{d}$, let $\mathcal{B}(\mathcal{R})$ be the Borel sets contained in $\mathcal{R}$, and let $\mathcal{A}$ be the $\delta$-ring of bounded Borel subsets of $\mathcal{R}$. 
Following [36], we consider a collection of real-valued random variables $L=\{L(A), A \in$ A) with the following properties:

- for every sequence $\left\{A_{n}\right\}$ of disjoint sets in $\mathcal{A}, L\left(A_{1}\right), \ldots, L\left(A_{n}\right), \ldots$ are independent random variables and $L\left(\bigcup_{n} A_{n}\right)=\sum_{n} L\left(A_{n}\right)$ almost surely (a.s.) provided that $\bigcup_{n} A_{n} \in \mathcal{A}$,

- for every $A$ in $\mathcal{A}, L(A)$ is infinitely divisible.

If $L$ has these properties, $L$ will be called a Lévy basis; cf. [2]. If $L(A) \geq 0$ for all $A \in \mathcal{A}$, $L$ is called a nonnegative Lévy basis.

For a random variable $X$, the logarithm of the characteristic function, $\log \mathrm{E}\left(\mathrm{e}^{\mathrm{i} v X}\right)$, will be called the cumulant function and will be denoted by $C(v, X)$. This notation has been used in [2], where the terminology of a Lévy basis was introduced. When $L$ is a Lévy basis, the cumulant function of $L(A)$ can, by the Lévy-Khintchine representation, be written as

$$
C(v, L(A))=\mathrm{i} v a(A)-\frac{1}{2} v^{2} b(A)+\int_{\mathbb{R}}\left(\mathrm{e}^{\mathrm{i} v r}-1-\mathrm{i} v r \mathbf{1}_{[-1,1]}(r)\right) U(\mathrm{~d} r, A),
$$

where $a$ is a $\sigma$-additive set function on $\mathcal{A}, b$ is a measure on $\mathscr{B}(\mathcal{R})$, and $U(\mathrm{~d} r, A)$ is a measure on $\mathscr{B}(\mathcal{R})$ for fixed $\mathrm{d} r$ and a Lévy measure on $\mathcal{B}(\mathbb{R})$ for each fixed $A \in \mathscr{B}(\mathcal{R})$, i.e. $U(\{0\}, A)=0$ and $\int_{\mathbb{R}}\left(1 \wedge r^{2}\right) U(\mathrm{~d} r, A)<\infty$, where ' $\wedge$ ' denotes the minimum. In fact, $U$ is a measure on $\mathscr{B}(\mathbb{R}) \times \mathscr{B}(\mathcal{R})$; cf. [36, Lemma 2.3]. This measure is referred to as the generalized Lévy measure and $L$ is said to have characteristic triplet $(a, b, U)$. If $b=0$ then $L$ is called a Lévy jump basis; if $U=0$ then $L$ is a Gaussian basis; see the examples below. A general Lévy basis $L$ can always be written as a sum of a Gaussian basis and an independent Lévy jump basis. Note that the term iva $(A)$ corresponds to a nonrandom shift of the values of $L$. The nonrandom shift may be included in the Gaussian component or in the jump component of the Lévy basis, or may be shared amongst them.

Let $|a|=a^{+}+a^{-}$. Then there exists a unique nonnegative measure $\mu$ on $\mathcal{B}(\mathcal{R})$ satisfying

$$
\mu(A)=|a|(A)+b(A)+\int_{\mathbb{R}}\left(1 \wedge r^{2}\right) U(\mathrm{~d} r, A) \quad \text { for } A \in \mathcal{A} ;
$$

cf. [36, Proposition 2.1, Definition 2.2]. We will call $\mu$ the control measure. In [36, Lemma 2.3] it was shown that the generalized Lévy measure $U$ factorizes as

$$
U(\mathrm{~d} r, \mathrm{~d} \eta)=V(\mathrm{~d} r, \eta) \mu(\mathrm{d} \eta),
$$

where $V(\mathrm{~d} r, \eta)$ is a Lévy measure for fixed $\eta$. Moreover, $a$ and $b$ are absolutely continuous with respect to $\mu$, i.e.

$$
a(\mathrm{~d} \eta)=\tilde{a}(\eta) \mu(\mathrm{d} \eta), \quad b(\mathrm{~d} \eta)=\tilde{b}(\eta) \mu(\mathrm{d} \eta),
$$

and, obviously, $|\tilde{a}|, \tilde{b} \leq 1 \mu$-a.s.

Let $L^{\prime}(\eta)$ be a random variable with the cumulant function

$$
C\left(v, L^{\prime}(\eta)\right)=\mathrm{i} v \tilde{a}(\eta)-\frac{1}{2} v^{2} \tilde{b}(\eta)+\int_{\mathbb{R}}\left(\mathrm{e}^{\mathrm{i} v r}-1-\mathrm{i} v r \mathbf{1}_{[-1,1]}(r)\right) V(\mathrm{~d} r, \eta) .
$$

Then, we obtain the representation

$$
C(v, L(A))=\int_{A} C\left(v, L^{\prime}(\eta)\right) \mu(\mathrm{d} \eta) .
$$


The random variables $L^{\prime}(\eta)$ will play an important role in the following and will be called spot variables. Note that $L^{\prime}(\eta)$ characterizes the behavior of $L$ at location $\eta$. For later use, note that if $\mathrm{E}\left(L^{\prime}(\eta)\right)$ and $\operatorname{var}\left(L^{\prime}(\eta)\right)$ exist then

$$
\begin{gathered}
\mathrm{E}\left(L^{\prime}(\eta)\right)=\tilde{a}(\eta)+\int_{[-1,1]^{C}} r V(\mathrm{~d} r, \eta), \\
\operatorname{var}\left(L^{\prime}(\eta)\right)=\tilde{b}(\eta)+\int_{\mathbb{R}} r^{2} V(\mathrm{~d} r, \eta) .
\end{gathered}
$$

By (1)-(3), it is no restriction in generality if, for modeling purposes, we only consider Lévy bases with characteristic triplet $(a, b, U)$ of the form

$$
\begin{aligned}
a(\mathrm{~d} \eta) & =\tilde{a}_{v}(\eta) v(\mathrm{~d} \eta), \\
b(\mathrm{~d} \eta) & =\tilde{b}_{v}(\eta) v(\mathrm{~d} \eta), \\
U(\mathrm{~d} r, \mathrm{~d} \eta) & =V_{v}(\mathrm{~d} r, \eta) v(\mathrm{~d} \eta),
\end{aligned}
$$

where $v$ is a nonnegative measure on $\mathscr{B}(\mathcal{R}), a_{v}: \mathcal{R} \rightarrow \mathbb{R}$ and $b_{v}: \mathcal{R} \rightarrow[0, \infty)$ are measurable functions, and $V_{v}(\mathrm{~d} r, \eta)$ is a Lévy measure for fixed $\eta$. The random variable satisfying (5), with $\mu$ replaced by $v$, will be denoted by $L_{v}^{\prime}(\eta)$. For simplicity, we write $L_{\mu}^{\prime}(\eta)=L^{\prime}(\eta)$, $\tilde{a}_{\mu}=\tilde{a}, \tilde{b}_{\mu}=\tilde{b}$, and $V_{\mu}(\mathrm{d} r, \eta)=V(\mathrm{~d} r, \eta)$. If $V_{v}(\cdot, \eta), \tilde{a}_{v}(\eta)$, and $\tilde{b}_{v}(\eta)$ do not depend on $\eta$, neither does the distribution of $L_{v}^{\prime}(\eta)$ and the Lévy basis $L$ is called $v$-factorizable. If, moreover, the measure $v$ is proportional to the Lebesgue measure, $L$ is called homogeneous and all the finite-dimensional distributions of $L$ are translation invariant.

Now let us consider the integration of a measurable function $f$ on $(\mathcal{R}, \mathcal{B}(\mathcal{R}))$ with respect to a Lévy basis $L$. The function $f$ is said to be integrable with respect to $L$ (cf. [36]) if there exists a sequence of simple functions $f_{n}$ converging to $f \mu$-almost everywhere and such that $\int_{A} f_{n} \mathrm{~d} L$ converges in probability as $n \rightarrow \infty$ for all $A \in \mathcal{B}(\mathcal{R})$. The limit is denoted $\int_{A} f \mathrm{~d} L$. The integral of a simple function $f_{n}=\sum_{j=1}^{k} x_{j} \mathbf{1}_{A_{j}^{n}}$ with respect to $L$ is defined in the following obvious manner:

$$
\int_{A} f_{n} \mathrm{~d} L=\sum_{j=1}^{k} x_{j} L\left(A \cap A_{j}^{n}\right) .
$$

The following lemma gives conditions for integrability and characterizes the distribution of the resulting integral.

Lemma 1. Let $f$ be a measurable function on $(\mathcal{R}, \mathcal{B}(\mathcal{R}))$, and let $L$ be a Lévy basis on $\mathcal{R}$ with characteristic triplet $(a, b, U)$. If

(i) $\int_{\mathcal{R}}|f(\eta)||a|(\mathrm{d} \eta)<\infty$,

(ii) $\int_{\mathcal{R}} f(\eta)^{2} b(\mathrm{~d} \eta)<\infty$, and

(iii) $\int_{\mathcal{R}} \int_{\mathbb{R}}|f(\eta) r| V(\mathrm{~d} r, \eta) \mu(\mathrm{d} \eta)<\infty$,

then the function $f$ is integrable with respect to $L$ and $\int_{\mathcal{R}} f \mathrm{~d} L$ is a well-defined random variable with the cumulant function

$$
\begin{aligned}
C\left(v, \int_{\mathcal{R}} f \mathrm{~d} L\right)= & \mathrm{i} v \int_{\mathcal{R}} f(\eta) a(\mathrm{~d} \eta)-\frac{1}{2} v^{2} \int_{\mathcal{R}} f(\eta)^{2} b(\mathrm{~d} \eta) \\
& +\int_{\mathcal{R}} \int_{\mathbb{R}}\left(\mathrm{e}^{\mathrm{i} f(\eta) v r}-1-\mathrm{i} f(\eta) v r \mathbf{1}_{[-1,1]}(r)\right) V(\mathrm{~d} r, \eta) \mu(\mathrm{d} \eta) .
\end{aligned}
$$


Proof. It suffices to check that the regularity conditions of [36, Theorem 2.7] are satisfied under the assumptions of Lemma 1. More specifically, we need to check that

(a) $\int_{\mathcal{R}}|h(f(\eta), \eta)| \mu(\mathrm{d} \eta)<\infty$,

(b) $\int_{\mathcal{R}} f(\eta)^{2} \tilde{b}(\eta) \mu(\mathrm{d} \eta)<\infty$, and

(c) $\int_{\mathcal{R}}\left(\int_{\mathbb{R}} \min \left\{1,(r f(\eta))^{2}\right\} V(\mathrm{~d} r, \eta)\right) \mu(\mathrm{d} \eta)<\infty$,

where

$$
h(u, \eta)=u \tilde{a}_{\tau}(\eta)+\int_{\mathbb{R}}(\tau(r u)-u \tau(r)) V(\mathrm{~d} r, \eta)
$$

Here

$$
\tau(r)=r \mathbf{1}_{[-1,1]}(r)+\frac{r}{|r|} \mathbf{1}_{[-1,1]^{C}}(r)
$$

and

$$
\tilde{a}_{\tau}(\eta)=\tilde{a}(\eta)+\int_{[-1,1]^{C}} \frac{r}{|r|} V(\mathrm{~d} r, \eta) .
$$

To prove (a), note that $|\tau(r u)| \leq|u r|$. Therefore,

$$
|h(f(\eta), \eta)| \leq\left|f(\eta) \tilde{a}_{\tau}(\eta)\right|+2 \int_{\mathbb{R}}|f(\eta) r| V(\mathrm{~d} r, \eta) .
$$

Using (i) and (iii) of Lemma 1, it follows that

$$
\int_{\mathcal{R}}|h(f(\eta), \eta)| \mu(\mathrm{d} \eta) \leq \int_{\mathcal{R}}|f(\eta) \tilde{a}(\eta)| \mu(\mathrm{d} \eta)+3 \int_{\mathcal{R}} \int_{\mathbb{R}}|f(\eta) r| V(\mathrm{~d} r, \eta) \mu(\mathrm{d} \eta)<\infty .
$$

Condition (b) is the same as (ii) and (c) follows from (iii) and the fact that

$$
\min \left\{1,(r f(\eta))^{2}\right\} \leq|r f(\eta)| .
$$

This completes the proof.

The conclusions of Lemma 1 hold under weaker assumptions; see [20, Proposition 5.6] or [36, Theorem 2.7]. The assumptions of Lemma 1 are simple to check and suffice for our purposes. Hellmund [20] also contains new selfcontained proofs of a number of other results concerning integration with respect to a Lévy basis.

Using (4), we can rewrite (9) as

$$
C\left(v, \int_{\mathcal{R}} f \mathrm{~d} L\right)=\int_{\mathcal{R}} C\left(v f(\eta), L^{\prime}(\eta)\right) \mu(\mathrm{d} \eta) .
$$

The logarithm of the Laplace transform of a random variable $X$ will be called the kumulant function and denoted by $K(v, X)=\log \mathrm{E}\left(\mathrm{e}^{-v X}\right)$ for $v \in \mathbb{R}$, in accordance with the notation used in [2]. If the kumulant function of the integral $\int_{\mathcal{R}} f \mathrm{~d} L$ exists then

$$
K\left(v, \int_{\mathcal{R}} f \mathrm{~d} L\right)=\int_{\mathcal{R}} K\left(v f(\eta), L^{\prime}(\eta)\right) \mu(\mathrm{d} \eta)
$$


Example 1. (Gaussian Lévy basis.) If $L$ is a Gaussian Lévy basis with characteristic triplet $(a, b, 0)$ then $L(A)$ is $N(a(A), b(A))$-distributed for each set $A \in \mathcal{A}$. If (6) and (7) hold, we obtain $L_{v}^{\prime}(\eta) \sim N\left(\tilde{a}_{v}(\eta), \tilde{b}_{v}(\eta)\right)$. Furthermore,

$$
C\left(v, \int_{\mathcal{R}} f \mathrm{~d} L\right)=\mathrm{i} v \int_{\mathcal{R}} f(\eta) a(\mathrm{~d} \eta)-\frac{1}{2} v^{2} \int_{\mathcal{R}} f(\eta)^{2} b(\mathrm{~d} \eta) .
$$

It follows that

$$
\int_{\mathcal{R}} f \mathrm{~d} L \sim N\left(\int_{\mathcal{R}} f(\eta) a(\mathrm{~d} \eta), \int_{\mathcal{R}} f(\eta)^{2} b(\mathrm{~d} \eta)\right) .
$$

The basis is $v$-factorizable when $\tilde{a}_{v}$ and $\tilde{b}_{v}$ are constant. A concrete example of a Gaussian Lévy basis is obtained by attaching independent Gaussian random variables $\left\{X_{i}\right\}$ to a locally finite sequence $\left\{\eta_{i}\right\}$ of fixed points and letting

$$
L(A)=\sum_{\eta_{i} \in A} X_{i}, \quad A \in \mathcal{A}
$$

Another example of a Gaussian Lévy basis is the white noise process; cf., e.g. [24, Section 1.3].

Example 2. (Poisson Lévy basis.) The simplest Lévy jump basis is the Poisson basis for which $L(A) \sim \operatorname{Po}(v(A))$, where $v$ is a nonnegative measure on $\mathcal{B}(\mathcal{R})$. Clearly, $L$ is a nonnegative Lévy basis. This basis has characteristic triplet $\left(\nu, 0, \delta_{1}(\mathrm{~d} r) v(\mathrm{~d} \eta)\right)$, where $\delta_{c}$ denotes the Dirac measure concentrated at $c$. Note that $\tilde{a}_{v}(\eta) \equiv 1$ and $V_{v}(\mathrm{~d} r, \eta)=\delta_{1}(\mathrm{~d} r)$. This basis is always $\nu$-factorizable. The random variable $L_{\nu}^{\prime}(\eta)$ has a $\operatorname{Po}(1)$ distribution.

Example 3. (Generalized G-Lévy basis.) A broad and versatile class of (nonnegative) Lévy jump bases are the so-called generalized $G$-Lévy bases with characteristic triplet of the form $(a, 0, U)$ depending on a nonnegative measure $v$ on $\mathcal{B}(\mathcal{R})$. The measures $a$ and $U$ satisfy (6) and (8) with

$$
V_{\nu}(\mathrm{d} r, \eta)=\mathbf{1}_{\mathbb{R}_{+}}(r) \frac{r^{-\alpha-1}}{\Gamma(1-\alpha)} \mathrm{e}^{-\theta(\eta) r} \mathrm{~d} r \quad \text { and } \quad \tilde{a}_{\nu}(\eta)=\int_{0}^{1} \frac{r^{-\alpha}}{\Gamma(1-\alpha)} \mathrm{e}^{-\theta(\eta) r} \mathrm{~d} r,
$$

where $\alpha \in(-\infty, 1), \theta: \mathcal{R} \rightarrow(0, \infty)$ is a measurable function, and $\Gamma$ denotes the gamma function. The class includes two important special cases: the gamma Lévy basis for $\alpha=$ 0 with $L_{v}^{\prime}(\eta) \sim \Gamma(1, \theta(\eta))$ and the inverse Gaussian Lévy basis for $\alpha=\frac{1}{2}$ with $L_{v}^{\prime}(\eta) \sim$ $I G(\sqrt{2}, \sqrt{2 \theta(\eta)})$. If the function $\theta$ is constant, $\theta(\eta)=\theta$, we find that $L(A) \sim G(\alpha, v(A), \theta)$, i.e. $L$ is a $G$-measure as defined in [5, Section 2].

The following theorem is a special case of the Lévy-Itô decomposition. This theorem will play a crucial role in the interpretation of some of the Lévy-driven Cox processes to be considered in the subsequent sections.

Theorem 1. Suppose that the Lévy basis L has no Gaussian part $(b=0)$ and that its generalized Lévy measure $U$ satisfies the following conditions:

- $U(\{(r, \eta)\})=0$ for all $(r, \eta) \in \mathbb{R} \times \mathcal{R}$ ( $U$ is diffuse),

- $\int_{[-1,1] \times A}|r| U(\mathrm{~d} r, \mathrm{~d} \eta)<\infty$ for all $A \in \mathcal{A}$. 
Then

$$
L(A)=a_{0}(A)+\int_{\mathbb{R}} r N(\mathrm{~d} r, A), \quad A \in \mathcal{A},
$$

where

$$
a_{0}(A)=a(A)-\int_{[-1,1]} r U(\mathrm{~d} r, A), \quad A \in \mathcal{A},
$$

and $N$ is a Poisson measure on $\mathbb{R} \times \mathcal{R}$ with intensity measure $U$.

The conditions of Theorem 1 are satisfied for a Poisson Lévy basis and a generalized $G$-Lévy basis if $v$ is a diffuse, locally finite measure on $\mathscr{B}(\mathcal{R})$.

\section{Cox processes}

Let $S$ be a Borel subset of $\mathbb{R}^{d}$, and suppose that $\{\Lambda(\xi): \xi \in S\}$ is a nonnegative random field which is almost surely integrable (with respect to the Lebesgue measure) on bounded Borel subsets of $S$. A point process $X$ on $S$ is a Cox process with the driving field $\Lambda$, if conditionally on $\Lambda, X$ is a Poisson process with intensity $\Lambda$; cf. [11], [12], and [31]. The driving measure $\Lambda_{\mathrm{M}}$ of the Cox process $X$ is defined by

$$
\Lambda_{\mathrm{M}}(B)=\int_{B} \Lambda(\xi) \mathrm{d} \xi, \quad B \in \mathscr{B}_{b}(S),
$$

where $\mathscr{B}_{b}(S)$ is the bounded Borel subsets of $S$.

In the following, the intensity function of $X$ will be denoted by $\rho(\xi)$ and, more generally, $\rho^{(n)}(\xi)$ is the $n$ th-order product density of $X$. It follows from the conditional structure of $X$ that $\rho^{(n)}$ can be computed from $\Lambda$ by

$$
\rho^{(n)}\left(\xi_{1}, \ldots, \xi_{n}\right)=\mathrm{E}\left(\prod_{i=1}^{n} \Lambda\left(\xi_{i}\right)\right), \quad \xi_{i} \in S
$$

(for a proof, using moment measures, see, e.g. [12]). A useful characteristic of a point process is the pair correlation function defined by

$$
g\left(\xi_{1}, \xi_{2}\right)=\frac{\rho^{(2)}\left(\xi_{1}, \xi_{2}\right)}{\rho^{(1)}\left(\xi_{1}\right) \rho^{(1)}\left(\xi_{2}\right)}, \quad \xi_{1}, \xi_{2} \in S,
$$

provided that $\rho^{(1)}\left(\xi_{i}\right)>0$ for $i=1,2$. (We let $g\left(\xi_{1}, \xi_{2}\right)=0$ if $\rho^{(1)}\left(\xi_{1}\right) \rho^{(1)}\left(\xi_{2}\right)=0$; cf. [31, p. 31].) Note that, for a Cox process, the pair correlation function can be calculated as

$$
g\left(\xi_{1}, \xi_{2}\right)=\frac{\mathrm{E}\left(\Lambda\left(\xi_{1}, \xi_{2}\right)\right)}{\mathrm{E}\left(\Lambda\left(\xi_{1}\right)\right) \mathrm{E}\left(\Lambda\left(\xi_{2}\right)\right)} .
$$

It can be shown that a Cox process is overdispersed relative to the Poisson process, i.e.

$$
\operatorname{var}(X(B)) \geq \mathrm{E}(X(B)),
$$

where $X(B)$ denotes the number of points from $X$ falling in $B$.

Examples of Cox processes include shot noise Cox processes (see [5], [28], and [49]) with a driving field of the form

$$
\Lambda(\xi)=\sum_{(r, \eta) \in \Phi} r k(\xi, \eta)
$$


where $k$ is a probability $\operatorname{kernel}(k(\cdot, \eta)$ is a probability density) and $\Phi$ is the atoms of a Poisson measure on $\mathbb{R}_{+} \times \mathcal{R}$, say. Concrete examples of probability kernels are the uniform kernel

$$
k(\xi, \eta)=\frac{1}{\omega_{d} R^{d}} \mathbf{1}_{[0, R]}(\|\xi-\eta\|),
$$

where $R>0$ and $\omega_{d}=\pi^{d / 2} / \Gamma(1+d / 2)$ is the volume of the unit ball in $\mathbb{R}^{d}$, and the Gaussian kernel

$$
k(\xi, \eta)=\frac{1}{\left(2 \pi \sigma^{2}\right)^{d / 2}} \exp \left(-\frac{\|\xi-\eta\|^{2}}{2 \sigma^{2}}\right),
$$

where $\sigma^{2}>0$. Another important class of Cox processes are the log Gaussian Cox processes (see [33]) driven by the exponential of a Gaussian field $\Psi$,

$$
\Lambda(\xi)=\exp (\Psi(\xi))
$$

\section{Lévy-driven Cox processes (LCPs)}

\subsection{Definition}

A point process $X$ on $S$ is called an LCP if $X$ is a Cox process with a driving field of the form

$$
\Lambda(\xi)=\int_{\mathcal{R}} k(\xi, \eta) L(\mathrm{~d} \eta), \quad \xi \in S,
$$

where $L$ is a nonnegative Lévy basis on $\mathcal{R}$. Furthermore, $k$ is a nonnegative function on $S \times \mathcal{R}$ such that $k(\xi, \cdot)$ is integrable with respect to $L$ for each $\xi \in S$ and $k(\cdot, \eta)$ is integrable with respect to the Lebesgue measure on $S$ for each $\eta \in \mathcal{R}$.

Note that, for each pair $(k, L)$, it is always possible to construct an associated pair $(\tilde{k}, \tilde{L})$ generating the same driving field $\Lambda$, where now $\tilde{k}(\cdot, \eta)$ is a probability kernel. We may simply let

$$
\tilde{k}(\xi, \eta)=\frac{k(\xi, \eta)}{\alpha(\eta)} \quad \text { and } \quad \tilde{L}(\mathrm{~d} \eta)=\alpha(\eta) L(\mathrm{~d} \eta)
$$

where

$$
\alpha(\eta)=\int_{S} k(\xi, \eta) \mathrm{d} \xi
$$

is assumed to be strictly positive. In the formulation and analysis of the models it is, however, not always convenient to restrict to probability kernels.

It is important to note that, from the nonnegativity of the Lévy basis $L$ and [12, Theorem 6.1.VI], we find that $L$ is equivalent to a random measure on $\mathcal{R}$. Thus, the measurability of $\Lambda$ defined in (12) follows from measurability of $k$ as a function of $\eta$ and $\xi$ and Tonelli's theorem. Therefore, $\Lambda$ is a well-defined random field and (under the condition of local integrability; see below) the driving measure $\int_{B} \Lambda(\xi) \mathrm{d} \xi, B \in \mathscr{B}_{b}(S)$, is also a well-defined random measure determined by the finite-dimensional distributions of $L$.

It will be assumed that the function $k$ and the Lévy basis $L$ have been chosen such that $\Lambda$ is almost surely locally integrable, i.e. $\int_{B} \Lambda(\xi) \mathrm{d} \xi<\infty$ with probability 1 for $B \in \mathscr{B}_{b}(S)$. A sufficient condition for the last property is that (cf. [31, Remark 5.1])

$$
\int_{B} \mathrm{E}(\Lambda(\xi)) \mathrm{d} \xi<\infty, \quad B \in \mathcal{B}_{b}(S) .
$$


If $L$ is factorizable then (13) is satisfied if the following conditions hold:

$$
\begin{gathered}
\int_{1}^{\infty} r V(\mathrm{~d} r)<\infty, \\
\int_{B} \int_{\mathcal{R}} k(\xi, \eta) \mu(\mathrm{d} \eta) \mathrm{d} \xi<\infty, \quad B \in \mathscr{B}_{b}(S) .
\end{gathered}
$$

\subsection{The $n$ th-order product densities of an LCP}

It is possible to derive a number of properties of LCPs, using the theory of Lévy bases presented in Section 2. Below, the $n$ th-order product densities, the generating functional, and the void probabilities of an LCP are considered. In Proposition 1, below, (complete) Bell polynomials, well known in combinatorics, are used; see [10].

Proposition 1. Suppose that

$$
\mathrm{E}\left(\int_{\mathcal{R}} k(\xi, \eta) L(\mathrm{~d} \eta)\right)^{n}<\infty
$$

and that

$$
\int_{\mathcal{R}} \int_{\mathbb{R}_{+}}(k(\xi, \eta) r)^{n} V(\mathrm{~d} r, \eta) \mu(\mathrm{d} \eta)<\infty \text { for all } \xi \in S .
$$

Then, the nth-order product density of an LCP is given by

$$
\rho^{(n)}\left(\xi_{1}, \ldots, \xi_{n}\right)=\frac{1}{2^{n} n !} \sum_{t \in T_{n}}\left(\prod_{j=1}^{n} t_{j}\right) B_{n}\left(\kappa_{1}(t), \ldots, \kappa_{n}(t)\right), \quad \xi_{1}, \ldots, \xi_{n} \in S,
$$

where $T_{n}$ denotes the set of all functions from $\{1, \ldots, n\}$ to $\{-1,1\}^{n}, B_{n}$ is the $n$th complete Bell polynomial evaluated at

$$
\kappa_{j}(t)=\int_{\mathcal{R}}\left(\sum_{i=1}^{n} t_{i} k\left(\xi_{i}, \eta\right)\right)^{j} \kappa_{j}\left(L^{\prime}(\eta)\right) \mu(\mathrm{d} \eta), \quad j=1, \ldots, n,
$$

and $\kappa_{j}\left(L^{\prime}(\eta)\right)$ is the jth kumulant moment of the spot variable $L^{\prime}(\eta)$.

Proof. First we rewrite $\rho^{(n)}\left(\xi_{1}, \ldots, \xi_{n}\right)=\mathrm{E}\left(\prod_{i=1}^{n} \Lambda\left(\xi_{i}\right)\right)$, using the polarization formula (cf. [15, p. 43])

$$
\mathrm{E}\left(\prod_{i=1}^{n} \Lambda\left(\xi_{i}\right)\right)=\frac{1}{2^{n} n !} \sum_{t \in T_{n}}\left(\prod_{i=1}^{n} t_{i}\right) \mathrm{E}\left(\sum_{i=1}^{n} t_{i} \Lambda\left(\xi_{i}\right)\right)^{n}
$$

The terms

$$
\mathrm{E}\left(\sum_{i=1}^{n} t_{i} \Lambda\left(\xi_{i}\right)\right)^{n}
$$

can be computed by evaluating the $n$th complete Bell polynomial in the first $n$ cumulants of $\sum_{i=1}^{n} t_{i} \Lambda\left(\xi_{i}\right)=\int_{\mathcal{R}} \sum_{i=1}^{n} t_{i} k\left(\xi_{i}, \eta\right) L(\mathrm{~d} \eta)$. Thus, we have

$$
\mathrm{E}\left(\sum_{i=1}^{n} t_{i} \Lambda\left(\xi_{i}\right)\right)^{n}=B_{n}\left(\kappa_{1}(t), \ldots, \kappa_{n}(t)\right)
$$


where $\kappa_{j}(t)$ is the $j$ th cumulant of

$$
\int_{\mathcal{R}} \sum_{i=1}^{n} t_{i} k\left(\xi_{i}, \eta\right) L(\mathrm{~d} \eta) .
$$

Under the assumptions of the proposition, $\kappa_{j}(t)$ can be calculated by differentiating (10) $j$ times with $f(\eta)=\sum_{i=1}^{n} t_{i} k\left(\xi_{i}, \eta\right)$. We obtain

$$
\kappa_{j}(t)=\int_{\mathcal{R}}\left(\sum_{i=1}^{n} t_{i} k\left(\xi_{i}, \eta\right)\right)^{j} \kappa_{j}\left(L^{\prime}(\eta)\right) \mu(\mathrm{d} \eta) .
$$

Note that

$$
\mathrm{E}\left(\int_{\mathcal{R}} \sum_{i=1}^{n} t_{i} k\left(\xi_{i}, \eta\right) L(\mathrm{~d} \eta)\right)^{j}<\infty
$$

and

$$
\int_{\mathcal{R}} \int_{\mathbb{R}_{+}}\left(\sum_{i=1}^{n} t_{i} k\left(\xi_{i}, \eta\right)\right)^{j} V(\mathrm{~d} r, \eta) \mu(\mathrm{d} \eta)<\infty, \quad j=1, \ldots, n,
$$

under the assumptions of the proposition. This completes the proof.

Corollary 1. Suppose that $k(\xi, \cdot)$ satisfies the assumptions of Lemma 1 for each $\xi \in S$. Then the intensity function of the LCP exists and is given by

$$
\rho(\xi)=\int_{\mathcal{R}} k(\xi, \eta) \mathrm{E}\left(L^{\prime}(\eta)\right) \mu(\mathrm{d} \eta) \quad \text { for all } \xi \in S .
$$

Furthermore, if

$$
\mathrm{E}\left(\int_{\mathcal{R}} k(\xi, \eta) L(\mathrm{~d} \eta)\right)^{2}<\infty
$$

and

$$
\int_{\mathcal{R}} \int_{\mathbb{R}}(k(\xi, \eta) r)^{2} V(\mathrm{~d} r, \eta) \mu(\mathrm{d} \eta)<\infty \quad \text { for each } \xi \in S,
$$

the pair correlation function of the process exists and is given by

$$
g(\xi, \zeta)=1+\frac{\int_{\mathcal{R}} k(\xi, \eta) k(\zeta, \eta) \operatorname{var}\left(L^{\prime}(\eta)\right) \mu(\mathrm{d} \eta)}{\rho(\xi) \rho(\zeta)} \text { for all } \xi, \zeta \in S .
$$

Proof. The result follows from Proposition 1, using the fact that the first and second complete Bell polynomials are given by $B_{1}(x)=x$ and $B_{2}\left(x_{1}, x_{2}\right)=x_{1}^{2}+x_{2}$. Also, recall that $\kappa_{1}\left(L^{\prime}(\eta)\right)=\mathrm{E}\left(L^{\prime}(\eta)\right)$ and $\kappa_{2}\left(L^{\prime}(\eta)\right)=\operatorname{var}\left(L^{\prime}(\eta)\right)$.

Corollary 2. (Stationary LCP.) Let $S=\mathcal{R}=\mathbb{R}^{d}$, and assume that $k$ is a homogeneous kernel in the sense that

$$
k(\xi, \eta)=k(\xi-\eta) \quad \text { for all } \xi, \eta \in \mathbb{R}^{d} .
$$

Let $\int k(\eta) \mathrm{d} \eta=\alpha$. Assume that $L$ is a homogeneous Lévy basis with control measure $\mu(\mathrm{d} \eta)=$ $c \mathrm{~d} \eta$ for some $c>0$. Then (15) and (16) take the following simplified form:

$$
\rho=c \mathrm{E}\left(L^{\prime}\right) \alpha, \quad g(\xi, \zeta)=1+\frac{\operatorname{var}\left(L^{\prime}\right)}{\left(\mathrm{E}\left(L^{\prime}\right)\right)^{2}} \frac{I_{k}(\zeta-\xi)}{c},
$$



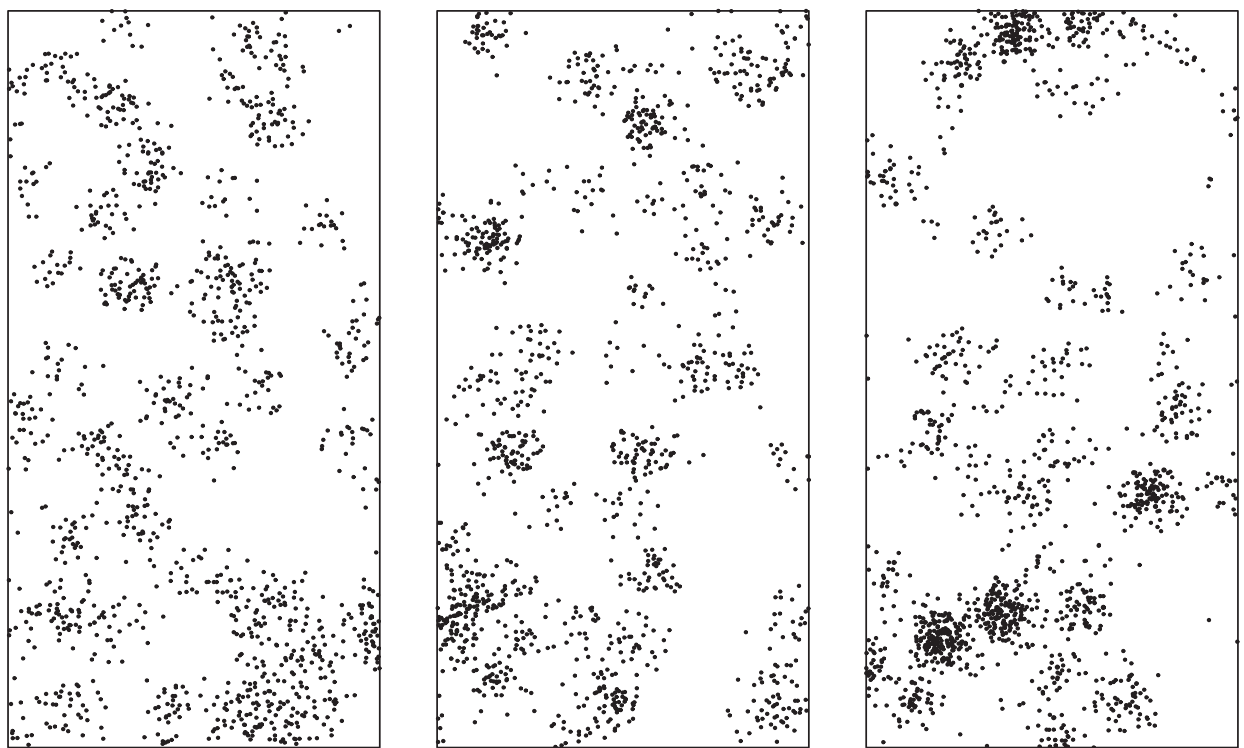

Figure 1: Examples of realizations of homogeneous LCPs with Poisson (left), gamma (middle), and inverse Gaussian (right) Lévy bases. For details, see the text.

where $I_{k}$ depends only on the kernel $k$,

$$
I_{k}(\zeta-\xi)=\int_{\mathbb{R}^{d}} \frac{k(\zeta-\xi+\eta) k(\eta)}{\alpha^{2}} \mathrm{~d} \eta
$$

Note that the fraction $\operatorname{var}\left(L^{\prime}\right) /\left(\mathrm{E}\left(L^{\prime}\right)\right)^{2}$ is equal to $1 / \mathrm{E}\left(L^{\prime}\right), 1$, and $\mathrm{E}\left(L^{\prime}\right)$ for the Poisson, gamma, and inverse Gaussian bases, respectively. The choice of the Lévy basis substantially changes the correlations in the LCP and the overall variability in the point pattern even when the corresponding LCPs are stationary and all other parameters of the model are the same. As an illustration, Figure 1 shows three stationary LCPs observed on a $[0,100] \times[0,200]$ window with $c=0.003, \mathrm{E}\left(L^{\prime}\right)=2$, and a Gaussian kernel obtained as 10 times kernel (11) with $\sigma=4$. The spot variable $L^{\prime}$ is distributed as $\mathrm{E}\left(L^{\prime}\right)$ times a $\mathrm{Po}(1)$-distributed variable, as a $\Gamma\left(1, \mathrm{E}\left(L^{\prime}\right)\right)$-distributed variable, and as an $I G\left(1,1 / \mathrm{E}\left(L^{\prime}\right)\right)$-distributed variable, respectively. From left to right in Figure 1, an increasing irregularity is clearly visible.

The distribution of a point process $X$ on $S$ can be characterized by the probability generating functional $G_{X}$. This functional is defined by

$$
G_{X}(u)=\mathrm{E}\left(\prod_{\xi \in X} u(\xi)\right)
$$

for functions $u: S \rightarrow[0,1]$ with $\{\xi \in S: u(\xi)<1\}$ bounded. As proved, for example, in [12], the probability generating functional of a Cox process can be computed by

$$
G_{X}(u)=\mathrm{E}\left(\exp \left(-\int_{S}(1-u(\xi)) \Lambda(\xi) \mathrm{d} \xi\right)\right) .
$$

Void probabilities can be calculated as

$$
v(B):=\mathrm{P}(X \cap B=\varnothing)=\mathrm{E}\left(\exp \left(-\int_{B} \Lambda(\xi) \mathrm{d} \xi\right)\right), \quad B \in \mathcal{B}_{b}(S) .
$$


Below, we give the expressions for $G_{X}$ and $v$ for an LCP.

Proposition 2. The probability generating functional of an LCP has the following form:

$$
\begin{aligned}
G_{X}(u)=\exp ( & -\int_{\mathbb{R}_{+}} \int_{\mathcal{R}}\left(1-\exp \left(-\int_{S}(1-u(\xi)) k(\xi, \eta) r \mathrm{~d} \xi\right)\right) U(\mathrm{~d} r, \mathrm{~d} \eta) \\
& \left.-\int_{\mathcal{R}} \int_{S}(1-u(\xi)) k(\xi, \eta) \mathrm{d} \xi a_{0}(\mathrm{~d} \eta)\right)
\end{aligned}
$$

while the void probabilities are given by

$$
\begin{aligned}
v(B)=\exp ( & -\int_{\mathbb{R}_{+}} \int_{\mathcal{R}}\left(1-\exp \left(-r \int_{B} k(\xi, \eta) \mathrm{d} \xi\right)\right) U(\mathrm{~d} r, \mathrm{~d} \eta) \\
& \left.-\int_{\mathcal{R}} \int_{B} k(\xi, \eta) \mathrm{d} \xi a_{0}(\mathrm{~d} \eta)\right), \quad B \in \mathcal{B}_{b}(S) .
\end{aligned}
$$

Proof. Since $\Lambda(\xi)$ is almost surely locally integrable,

$$
\int_{S}(1-u(\xi)) \Lambda(\xi) \mathrm{d} \xi \leq \int_{S} \mathbf{1}_{\operatorname{supp}(1-u)}(\xi) \Lambda(\xi) \mathrm{d} \xi<\infty
$$

is a well-defined nonnegative random variable and its kumulant transform exists. (In (17) the support of the function $1-u$ is denoted $\operatorname{supp}(1-u)$.) Using the key relation (10) for the kumulant function, we obtain

$$
\begin{aligned}
\log \left(G_{X}(u)\right)= & \log \left(\mathrm{E}\left(\exp \left(-\int_{S}(1-u(\xi)) \Lambda(\xi) \mathrm{d} \xi\right)\right)\right) \\
= & K\left(1, \int_{S}(1-u(\xi)) \Lambda(\xi) \mathrm{d} \xi\right) \\
= & K\left(1, \int_{S}(1-u(\xi)) \int_{\mathcal{R}} k(\xi, \eta) L(\mathrm{~d} \eta) \mathrm{d} \xi\right) \\
= & K\left(1, \int_{\mathcal{R}}\left(\int_{S}(1-u(\xi)) k(\xi, \eta) \mathrm{d} \xi\right) L(\mathrm{~d} \eta)\right) \\
= & \int_{\mathcal{R}} K\left(\int_{S}(1-u(\xi)) k(\xi, \eta) \mathrm{d} \xi, L^{\prime}(\eta)\right) \mu(\mathrm{d} \eta) \\
= & -\int_{\mathcal{R}} \int_{S}(1-u(\xi)) k(\xi, \eta) \mathrm{d} \xi a_{0}(\mathrm{~d} \eta) \\
& +\int_{\mathcal{R}} \int_{\mathbb{R}_{+}}\left(\exp \left(-\int_{S}(1-u(\xi)) k(\xi, \eta) r \mathrm{~d} \xi\right)-1\right) V(\mathrm{~d} r, \eta) \mu(\mathrm{d} \eta) .
\end{aligned}
$$

The result for the void probabilities is obtained by choosing $u(\xi)=\mathbf{1}_{B^{c}}(\xi)$. This completes the proof.

\subsection{Mixing properties}

The following proposition gives conditions for stationarity and mixing of an LCP. Mixing and ergodicity are important, for example, for establishing the consistency of model parameter estimates, including nonparametric estimates of the $n$ th-order product density $\rho^{(n)}$ and the pair correlation function $g$. Mixing [12, Definition 10.3.I] implies ergodicity [12, p. 341]. The case of an LCP with $G$-Lévy basis has been treated in [5, Proposition 2.2]. 
Proposition 3. Let $S=\mathcal{R}=\mathbb{R}^{d}$, and assume that the Lévy basis L and the kernel $k$ are homogeneous. Then, an LCP with driving field $\Lambda$ of the form (12) is stationary and mixing.

Proof. Note that a Cox process is stationary and mixing if and only if the driving field of the Cox process has the same property [12, Proposition 10.3.VII]. Using the assumptions of the proposition, it is easily seen that $\left\{\Lambda(\xi+x): \xi \in \mathbb{R}^{d}\right\}$ has the same distribution as $\left\{\Lambda(\xi): \xi \in \mathbb{R}^{d}\right\}$ for all $x \in \mathbb{R}^{d}$.

According to [12, Proposition 10.3.VI(a)], $\Lambda$ is mixing if and only if

$$
\mathrm{L}_{\Lambda}\left[h_{1}+T_{x} h_{2}\right] \rightarrow \mathrm{L}_{\Lambda}\left[h_{1}\right] \mathrm{L}_{\Lambda}\left[h_{2}\right] \quad \text { as }\|x\| \rightarrow \infty \text {. }
$$

Here, $h_{1}$ and $h_{2}$ are arbitrary nonnegative bounded functions on $\mathbb{R}^{d}$ of bounded support, $\mathrm{L}_{\Lambda}$ is the Laplace functional defined by

$$
\mathrm{L}_{\Lambda}[h]=\mathrm{E}\left(\exp \left(-\int_{\mathbb{R}^{d}} h(\xi) \Lambda(\xi) \mathrm{d} \xi\right)\right)
$$

and $T_{x} h(\xi)=h(\xi+x), \xi, x \in \mathbb{R}^{d}$. We obtain

$$
\begin{aligned}
\mathrm{L}_{\Lambda}\left[h_{1}\right. & \left.+T_{x} h_{2}\right] \\
& =\mathrm{E}\left(\exp \left(-\int_{\mathbb{R}^{d}} \int_{\mathbb{R}^{d}}\left(h_{1}(\xi)+h_{2}(\xi+x)\right) k(\xi-\eta) L(\mathrm{~d} \eta) \mathrm{d} \xi\right)\right) \\
& =\mathrm{E}\left(\exp \left(-\int_{\mathbb{R}^{d}}\left(\int_{\mathbb{R}^{d}} h_{1}(\xi) k(\xi-\eta) \mathrm{d} \xi+\int_{\mathbb{R}^{d}} h_{2}(\xi) k(\xi-\eta-x) \mathrm{d} \xi\right) L(\mathrm{~d} \eta)\right)\right) \\
& =\mathrm{E}\left(\exp \left(-\int_{\mathbb{R}^{d}} \tilde{h}_{1}(\eta) L(\mathrm{~d} \eta)\right) \exp \left(-\int_{\mathbb{R}^{d}} \tilde{h}_{2}(\eta+x) L(\mathrm{~d} \eta)\right)\right),
\end{aligned}
$$

where

$$
\tilde{h}_{i}(\eta)=\int_{\mathbb{R}^{d}} h_{i}(\xi) k(\xi-\eta) \mathrm{d} \xi .
$$

If $k$ has bounded support then we can find a $C>0$ such that, for $\|x\|>C$,

$$
\left\{\eta \in \mathbb{R}^{d}: \tilde{h}_{1}(\eta)>0\right\} \cap\left\{\eta \in \mathbb{R}^{d}: \tilde{h}_{2}(\eta+x)>0\right\}=\varnothing .
$$

It follows that, for $\|x\|>C$,

$$
\begin{aligned}
\mathrm{L}_{\Lambda}\left[h_{1}+T_{x} h_{2}\right] & =\mathrm{E}\left(\exp \left(-\int_{\mathbb{R}^{d}} \tilde{h}_{1}(\eta) L(\mathrm{~d} \eta)\right)\right) \mathrm{E}\left(\exp \left(-\int_{\mathbb{R}^{d}} \tilde{h}_{2}(\eta+x) L(\mathrm{~d} \eta)\right)\right) \\
& =\mathrm{L}_{\Lambda}\left[h_{1}\right] \mathrm{L}_{\Lambda}\left[h_{2}\right],
\end{aligned}
$$

since $L$ is independently scattered. If $k$ does not have bounded support, we define a series of functions with bounded support

$$
k_{n}(\xi-\eta)=k(\xi-\eta) \mathbf{1}_{[0, n)}(\|\xi-\eta\|), \quad n=1,2, \ldots,
$$

that converges monotonically from below to $k$. It follows that $\tilde{h}_{i, n}$ defined by

$$
\tilde{h}_{i, n}(\eta)=\int_{\mathbb{R}^{d}} h_{i}(\xi) k_{n}(\xi-\eta) \mathrm{d} \xi
$$


converges monotonically from below to $\tilde{h}_{i}(\eta)$ and, for fixed $n$, we can find a $C_{n}$ such that, for $\|x\|>C_{n}$,

$$
\begin{aligned}
& \mathrm{E}\left(\exp \left(-\int_{\mathbb{R}^{d}} \tilde{h}_{1, n}(\eta) L(\mathrm{~d} \eta)\right) \exp \left(-\int_{\mathbb{R}^{d}} \tilde{h}_{2, n}(\eta+x) L(\mathrm{~d} \eta)\right)\right) \\
& \quad=\mathrm{E}\left(\exp \left(-\int_{\mathbb{R}^{d}} \tilde{h}_{1, n}(\eta) L(\mathrm{~d} \eta)\right)\right) \mathrm{E}\left(\exp \left(-\int_{\mathbb{R}^{d}} \tilde{h}_{2, n}(\eta+x) L(\mathrm{~d} \eta)\right)\right) .
\end{aligned}
$$

Using the reasoning just after [12, Proposition 10.3.VI], it follows that

$$
\mathrm{L}_{\Lambda}\left[h_{1}+T_{x} h_{2}\right] \rightarrow \mathrm{L}_{\Lambda}\left[h_{1}\right] \mathrm{L}_{\Lambda}\left[h_{2}\right]
$$

for the original functions $h_{1}$ and $h_{2}$. This completes the proof.

\subsection{Examples of LCPs}

4.4.1. Shot noise Cox processes with random noise. Under the assumptions of Theorem 1, the driving field of an LCP takes the form

$$
\Lambda(\xi)=\int_{\mathcal{R}} k(\xi, \eta) a_{0}(\mathrm{~d} \eta)+\sum_{(r, \eta) \in \Phi} r k(\xi, \eta),
$$

where $\Phi$ is the atoms of a Poisson measure on $\mathbb{R}_{+} \times \mathcal{R}$ with intensity measure $U$. An LCP $X$ with such a driving field is distributed as a superposition $X_{1} \cup X_{2}$, where $X_{1}$ and $X_{2}$ are independent, $X_{1}$ is a Poisson point process with intensity function

$$
\rho_{1}(\xi)=\int_{\mathcal{R}} k(\xi, \eta) a_{0}(\mathrm{~d} \eta)
$$

and $X_{2}$ is a shot noise Cox process as defined in [28] with driving field

$$
\Lambda_{2}(\xi)=\sum_{(r, \eta) \in \Phi} r k(\xi, \eta)
$$

An LCP with driving field $\Lambda$ of the form (18) is therefore a shot noise Cox process (SNCP) with additional random noise. Simulation of the associated Lévy basis can be performed, using the algorithm introduced in [16], if $L$ is factorizable, otherwise the algorithm developed in [50] may be used (see also [46]). A third option is the method used in [28]. An overview of available methods of simulating Lévy processes can be found in [39].

For $a_{0} \equiv 0$, we obtain the familiar SNCPs. In [28] three specific examples of stationary SNCPs are considered. Using the notion of a Lévy basis, they are specified by $U(\mathrm{~d} r, \mathrm{~d} \eta)=$ $V(\mathrm{~d} r, \eta) v(\mathrm{~d} \eta)$, where $v(\mathrm{~d} \eta) \propto \mathrm{d} \eta$, and one of the following conditions.

- $V$ is concentrated in a single point $c>0$, i.e. $V(\mathrm{~d} r)=\delta_{c}(\mathrm{~d} r)$. If $c=1$, the corresponding Lévy basis is Poisson. If $c \neq 1, L(A) \sim c \operatorname{Po}(v(A))$. LCPs of this type are the wellknown Matérn cluster process [25] and the Thomas process [42].

- $V((0, \infty))<\infty$. In this case, $\Phi$ can be represented as a marked Poisson point process. Examples of LCPs with such a Lévy basis are the Neyman-Scott processes; cf. [34].

$$
V(\mathrm{~d} r)=\mathbf{1}_{\mathbb{R}_{+}}(r) \frac{r^{-\alpha-1}}{\Gamma(1-\alpha)} \mathrm{e}^{-\theta r} \mathrm{~d} r
$$

corresponding to a $G$-Lévy basis. The resulting LCP is a so-called shot noise $G$-Cox process [5]. 


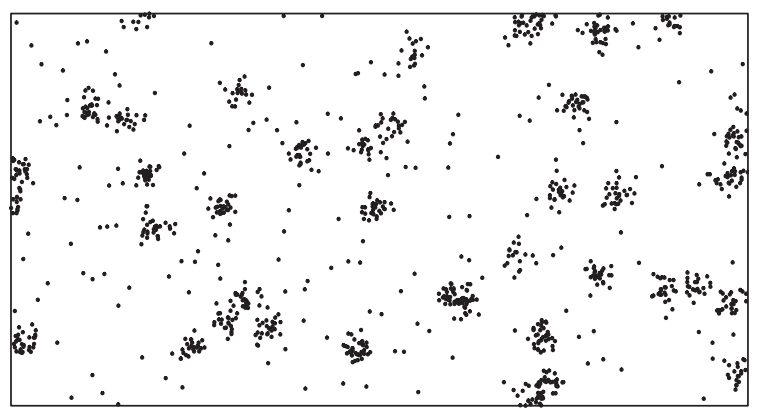

FIGURE 2: Example of an SNCP with extra noise. For details, see the text.

In Figure 2 we show an example of an SNCP with a homogeneous Poisson process $\left(a_{0}\right.$ is proportional to the Lebesgue measure) with additional random noise. More precisely, the process $X=X_{1} \cup X_{2}$ is defined on [0,200] $\times[0,100], X_{1}$ is a Poisson process with intensity 0.01 , and $X_{2}$ is an SNCP with Gaussian kernel (11) with $\sigma=2$ and an intensity measure $U$ of the form $U(\mathrm{~d} r, \mathrm{~d} \eta)=\delta_{25}(r) 0.0025 \mathrm{~d} \eta$. The process $X_{2}$ is thereby a Thomas process.

4.4.2. LCPs driven by smoothed discrete random fields. We suppose that $\left\{\eta_{i}\right\}$ is a locally finite sequence of fixed points, and let

$$
L(A)=\sum_{\eta_{i} \in A} X_{i}
$$

where $\left\{X_{i}\right\}$ is a sequence of independent and identically distributed nonnegative random variables with infinitely divisible distribution. If, for instance, $X_{i}$ is gamma or inverse Gaussian distributed then $L$ is a special case of a gamma or inverse Gaussian Lévy basis, respectively. The driving intensity of the associated LCP will take the form

$$
\Lambda(\xi)=\sum_{\eta_{i}} k\left(\xi, \eta_{i}\right) X_{i}
$$

\section{Log Lévy-driven Cox processes (LLCPs)}

\subsection{Definition}

A point process $X$ on $S$ is called an LLCP if $X$ is a Cox process with intensity field of the form

$$
\Lambda(\xi)=\exp \left(\int_{\mathcal{R}} k(\xi, \eta) L(\mathrm{~d} \eta)\right),
$$

where $L$ is a Lévy basis and $k$ is a kernel such that $k(\xi, \cdot)$ is integrable with respect to $L$ for each $\xi \in S, k(\cdot, \eta)$ is integrable with respect to the Lebesgue measure on $S$ for each $\eta \in \mathcal{R}$, and $\Lambda$ is almost surely locally integrable.

Since the driving intensity field of an LLCP is always nonnegative because of the exponential function, we can generally use kernels and Lévy bases which also have negative values. Moreover, using the Lévy-Khintchine representation, (1), we see that each Lévy basis $L$ is equal to a sum of two independent parts-a Lévy jump part (let us denote it by $L_{\mathrm{J}}$ ) and a Gaussian part (let us denote it by $L_{\mathrm{G}}$ ). Thus, we can represent the driving intensity of an LLCP as a product of two independent driving fields:

$$
\Lambda(\xi)=\exp \left(\int_{\mathcal{R}} k(\xi, \eta) L_{\mathrm{J}}(\mathrm{d} \eta)\right) \exp \left(\int_{\mathcal{R}} k(\xi, \eta) L_{\mathrm{G}}(\mathrm{d} \eta)\right)=\Lambda_{\mathrm{J}}(\xi) \Lambda_{\mathrm{G}}(\xi)
$$


If $L_{\mathrm{J}} \equiv 0, \Lambda$ is the driving field of a $\log$ Gaussian Cox process [9], [33]; if $L_{\mathrm{G}} \equiv 0, \Lambda$ is, under regularity conditions, the driving field of a log SNCP (see the examples in Section 5.4, below).

Because of the exponential function in the definition of $\Lambda(\xi)$, stronger conditions on $k$ and $L$ are needed in order to ensure that $\Lambda$ is almost surely locally integrable. A sufficient condition is that the kumulant transform $K\left(-k(\xi, \eta), L^{\prime}(\eta)\right)$ exists for all $\xi \in S$ and $\eta \in \mathcal{R}$, and that

$$
\int_{B} \exp \left(\int_{\mathcal{R}} K\left(-k(\xi, \eta), L^{\prime}(\eta)\right) \mu(\mathrm{d} \eta)\right) \mathrm{d} \xi<\infty \text { for all } B \in \mathscr{B}_{b}(S) .
$$

This result follows from the definition of the kumulant function and from the key relation (10) for the kumulant transform. In particular, we use

$$
\mathrm{E}(\Lambda(\xi))=\exp \left(K\left(1,-\int_{\mathcal{R}} k(\xi, \eta) L(\mathrm{~d} \eta)\right)\right)=\exp \left(\int_{\mathcal{R}} K\left(-k(\xi, \eta), L^{\prime}(\eta)\right) \mu(\mathrm{d} \eta)\right) .
$$

Note that

$$
\begin{aligned}
K\left(-k(\xi, \eta), L^{\prime}(\eta)\right)= & k(\xi, \eta) \tilde{a}(\eta)+\frac{1}{2} k(\xi, \eta)^{2} \tilde{b}(\eta) \\
& +\int_{\mathbb{R}}\left(\mathrm{e}^{k(\xi, \eta) r}-1-k(\xi, \eta) r \mathbf{1}_{[-1,1]}(r)\right) V(\mathrm{~d} r, \eta) .
\end{aligned}
$$

If $L$ is factorizable then (20) is satisfied if either there exist $B>0, C>0$, and $D>0$ such that

$$
\begin{gathered}
|k(\xi, \eta)| \leq C \quad \text { for all } \xi \in S, \eta \in \mathcal{R}, \\
\int_{\mathcal{R}}|k(\xi, \eta)|^{i} \mu(\mathrm{d} \eta)<B D^{i}, \quad i=1,2, \ldots, \xi \in S, \\
\int_{\mathbb{R}}\left(\mathrm{e}^{(C \vee D)|r|}-1-(C \vee D)|r| \mathbf{1}_{[-1,1]}(r)\right) V(\mathrm{~d} r)<\infty,
\end{gathered}
$$

or there exist $C>0$ and $R>0$ such that

$$
\begin{gathered}
|k(\xi, \eta)| \leq C \text { for all } \xi \in S, \eta \in \mathcal{R}, \\
k(\xi, \eta)=0 \text { for }\|\xi-\eta\|>R, \\
\mu \text { is locally finite, } \\
\int_{\mathbb{R}}\left(\mathrm{e}^{C|r|}-1-C|r| \mathbf{1}_{[-1,1]}(r)\right) V(\mathrm{~d} r)<\infty .
\end{gathered}
$$

Note that (21) and (22) are satisfied for the Gaussian kernel if $\mu$ is the Lebesgue measure, while (24) and (25) hold for the uniform kernel. In the case of a purely Gaussian basis, (22) is needed only for $i=2$ and conditions (23) and (26) are trivially satisfied since $V \equiv 0$.

\subsection{The $\boldsymbol{n}$-th order product densities of an LLCP}

The $n$ th-order product densities of LLCPs are easily derived, using Lévy theory.

Proposition 4. The nth-order product density is given by

$$
\rho^{(n)}\left(\xi_{1}, \ldots, \xi_{n}\right)=\exp \left(\int_{\mathcal{R}} K\left(-\sum_{i=1}^{n} k\left(\xi_{i}, \eta\right), L^{\prime}(\eta)\right) \mu(\mathrm{d} \eta)\right), \quad \xi_{1}, \ldots, \xi_{n} \in S,
$$

provided that the right-hand side exists. 
Proof. The formula follows directly from the definition of the kumulant function and from the key relation (10). We obtain

$$
\begin{aligned}
\rho^{(n)}\left(\xi_{1}, \ldots, \xi_{n}\right) & =\mathrm{E}\left(\prod_{i=1}^{n} \Lambda\left(\xi_{i}\right)\right) \\
& =\mathrm{E}\left(\exp \left(\sum_{i=1}^{n} \int_{\mathcal{R}} k\left(\xi_{i}, \eta\right) L(\mathrm{~d} \eta)\right)\right) \\
& =\exp \left(K\left(1,-\sum_{i=1}^{n} \int_{\mathcal{R}} k\left(\xi_{i}, \eta\right) L(\mathrm{~d} \eta)\right)\right) \\
& =\exp \left(\int_{\mathcal{R}} K\left(-\sum_{i=1}^{n} k\left(\xi_{i}, \eta\right), L^{\prime}(\eta)\right) \mu(\mathrm{d} \eta)\right)
\end{aligned}
$$

Corollary 3. The intensity function of an LLCP X is given by

$$
\rho(\xi)=\exp \left(\int_{\mathcal{R}} K\left(-k(\xi, \eta), L^{\prime}(\eta)\right) \mu(\mathrm{d} \eta)\right),
$$

provided that the right-hand side exists. When the second-order product density exists, the pair correlation function of an LLCP takes the following form:

$$
\begin{aligned}
g(\xi, \zeta)= & \exp \left(\int _ { \mathcal { R } } \left[K\left(-k(\xi, \eta)-k(\zeta, \eta), L^{\prime}(\eta)\right)\right.\right. \\
& \left.\left.-K\left(-k(\xi, \eta), L^{\prime}(\eta)\right)-K\left(-k(\zeta, \eta), L^{\prime}(\eta)\right)\right] \mu(\mathrm{d} \eta)\right) \\
= & \exp \left(\int_{\mathcal{R}} k(\xi, \eta) k(\zeta, \eta) b(\mathrm{~d} \eta)\right. \\
& \left.\quad+\int_{\mathcal{R}} \int_{\mathbb{R}}\left(\mathrm{e}^{(k(\xi, \eta)+k(\zeta, \eta)) r}-\mathrm{e}^{k(\xi, \eta) r}-\mathrm{e}^{k(\zeta, \eta) r}+1\right) V(\mathrm{~d} r, \eta) \mu(\mathrm{d} \eta)\right) .
\end{aligned}
$$

Corollary 4. (Stationary LLCP.) Let $S=\mathcal{R}=\mathbb{R}^{d}$. Assume that $k$ is a homogeneous kernel and that $L$ is a homogeneous Lévy basis with $\mu(\mathrm{d} \eta)=c \mathrm{~d} \eta$ for some $c>0$. Then,

$$
\rho=\exp \left(c \int_{\mathbb{R}^{d}} K\left(-k(\eta), L^{\prime}\right) \mathrm{d} \eta\right)
$$

and

$$
\begin{aligned}
& g(\xi, \zeta)=\exp \left(\tilde{b} c \int_{\mathbb{R}^{d}} k(\xi-\zeta+\eta) k(\eta) \mathrm{d} \eta\right. \\
& \left.\quad+c \int_{\mathbb{R}^{d}} \int_{\mathbb{R}}\left(\mathrm{e}^{(k(\xi-\zeta+\eta)+k(\eta)) r}-\mathrm{e}^{k(\xi-\zeta+\eta) r}-\mathrm{e}^{k(\eta) r}+1\right) V(\mathrm{~d} r) \mathrm{d} \eta\right) .
\end{aligned}
$$

\subsection{Mixing properties}

Proposition 5. Let $S=\mathcal{R}=\mathbb{R}^{d}$, and assume that the Lévy basis $L$ is homogeneous and that the kernel $k$ is homogeneous. Then an LLCP with driving field of the form (19) is stationary and mixing. 
Proof. As in the proof of Proposition 3, we immediately obtain the stationarity. The method of proving mixing has to be modified compared to the one used in Proposition 3. First, rewrite

$$
\begin{aligned}
\mathrm{L}_{\Lambda}\left[h_{1}+T_{x} h_{2}\right]= & \mathrm{E}\left(\exp \left(-\int_{\mathbb{R}^{d}} h_{1}(\xi) \exp \left(\int_{\mathbb{R}^{d}} k(\xi-\eta) L(\mathrm{~d} \eta)\right) \mathrm{d} \xi\right)\right. \\
& \left.\times \exp \left(-\int_{\mathbb{R}^{d}} h_{2}(\xi+x) \exp \left(\int_{\mathbb{R}^{d}} k(\xi-\eta) L(\mathrm{~d} \eta)\right) \mathrm{d} \xi\right)\right) \\
= & \mathrm{E}\left(A B_{x}\right),
\end{aligned}
$$

say. If $k$ has bounded support, $A$ and $B_{x}$ will be independent if $\|x\|$ is large enough. If $k$ does not have bounded support, we use a series of functions $k_{n}$ with bounded support that converges to $k$. To be precise, let, as in Proposition 3,

$$
k_{n}(u)=k(u) \mathbf{1}_{[0, n)}(\|u\|), \quad u \in \mathbb{R}^{d},
$$

$n=1,2, \ldots$ We have $k_{n} \rightarrow k$ and $\left|k_{n}\right| \leq|k|$. Now, let

$$
A_{n}=\exp \left(-\int_{\mathbb{R}^{d}} h_{1}(\xi) \exp \left(\int_{\mathbb{R}^{d}} k_{n}(\xi-\eta) L(\mathrm{~d} \eta)\right) \mathrm{d} \xi\right)
$$

and

$$
B_{x, n}=\exp \left(-\int_{\mathbb{R}^{d}} h_{2}(\xi+x) \exp \left(\int_{\mathbb{R}^{d}} k_{n}(\xi-\eta) L(\mathrm{~d} \eta)\right) \mathrm{d} \xi\right) .
$$

Note that $0 \leq A, A_{n}, B_{x}, B_{x, n} \leq 1$. Now, consider the following inequality

$$
\begin{aligned}
&\left|\mathrm{E}\left(A B_{x}\right)-\mathrm{E}(A) \mathrm{E}\left(B_{x}\right)\right| \\
& \leq\left|\mathrm{E}\left(A B_{x}\right)-\mathrm{E}\left(A B_{x, n}\right)\right|+\left|\mathrm{E}\left(A B_{x, n}\right)-\mathrm{E}\left(A_{n} B_{x, n}\right)\right| \\
&+\left|\mathrm{E}\left(A_{n} B_{x, n}\right)-\mathrm{E}\left(A_{n}\right) \mathrm{E}\left(B_{x, n}\right)\right|+\left|\mathrm{E}\left(A_{n}\right) \mathrm{E}\left(B_{x, n}\right)-\mathrm{E}\left(A_{n}\right) \mathrm{E}\left(B_{x}\right)\right| \\
&+\left|\mathrm{E}\left(A_{n}\right) \mathrm{E}\left(B_{x}\right)-\mathrm{E}(A) \mathrm{E}\left(B_{x}\right)\right| \\
&= \delta_{1 x n}+\delta_{2 x n}+\delta_{3 x n}+\delta_{4 x n}+\delta_{5 x n},
\end{aligned}
$$

say. Let us evaluate each of these five terms. Using the facts that $0 \leq A \leq 1$ and $L$ is homogeneous, we obtain

$$
\begin{aligned}
\delta_{1 x n} & =\left|\mathrm{E}\left(A\left(B_{x}-B_{x, n}\right)\right)\right| \\
& \leq \mathrm{E}\left(A\left|B_{x}-B_{x, n}\right|\right) \\
& \leq \mathrm{E}\left(\left|B_{x}-B_{x, n}\right|\right) \\
& =\mathrm{E}\left(\left|B_{0}-B_{0, n}\right|\right) .
\end{aligned}
$$

Now, since $k_{n} \rightarrow k$ and $\left|k_{n}\right| \leq|k|$, where $k$ is $L$-integrable, it follows that

$$
\int_{\mathbb{R}^{d}} k_{n}(\xi-\eta) L(\mathrm{~d} \eta) \rightarrow \int_{\mathbb{R}^{d}} k(\xi-\eta) L(\mathrm{~d} \eta) \quad \text { a.s. }
$$

We can therefore find $n_{1}$ (not dependent on $x$ ) such that, for $n \geq n_{1}, \delta_{1 x n} \leq \varepsilon$, say. Using the same type of arguments, we can find $n_{2}, n_{4}$, and $n_{5}$ such that, for $n \geq n_{i}, \delta_{i x n} \leq \varepsilon, i=2,4,5$. Now choose a fixed $n \geq \max \left(n_{1}, n_{2}, n_{4}, n_{5}\right)$ and consider

$$
\delta_{3 x n}=\left|\mathrm{E}\left(A_{n} B_{x, n}\right)-\mathrm{E}\left(A_{n}\right) \mathrm{E}\left(B_{x, n}\right)\right| .
$$

Using the previous results for bounded functions of bounded support, we finally find a constant, $C>0$, such that, for $x$ with $\|x\|>C$, we have $\delta_{3 x n} \leq \varepsilon$. This completes the proof. 


\subsection{Examples of LLCPs}

5.4.1. Log shot noise Cox processes (LSNCPs). Under the assumptions of Theorem 1, the driving field of an LLCP takes the form

$$
\Lambda(\xi)=\exp \left(d(\xi)+\sum_{(r, \eta) \in \Phi} r k(\xi, \eta)\right),
$$

where $d(\xi)$ is a deterministic function and $\Phi$ is the atoms of a Poisson measure on $\mathbb{R} \times \mathcal{R}$ with intensity measure $U$. Such a process is called a log shot noise Cox process (LSNCP).

It is important to realize that SNCPs and LSNCPs are quite different model classes. An SNCP $X$ with a driving field of the form

$$
\Lambda(\xi)=\sum_{(r, \eta) \in \Phi} r k(\xi, \eta)
$$

is a superposition of independent Poisson processes $X_{(r, \eta)},(r, \eta) \in \Phi$, where $X_{(r, \eta)}$ has intensity function $r k(\cdot, \eta)$. (The process $\{\eta:(r, \eta) \in \Phi\}$ is usually called the center process (although it is not necessarily locally finite) while $X_{(r, \eta)}$ is called a cluster around $\eta$.) The presence of a particular cluster $X_{(r, \eta)}$ will not affect the presence of the other clusters.

In contrast to this, the driving field of an LSNCP takes the form

$$
\Lambda(\xi)=\exp (d(\xi)) \prod_{(r, \eta) \in \Phi} \exp (r k(\xi, \eta)) .
$$

A cluster $X_{(r, \eta)}$ with negative, numerically large values of $r k(\cdot, \eta)$ will very likely contain zero points and, moreover, wipe out points from other clusters in the neighborhood of $\eta$. In the resulting point pattern, empty holes may therefore be present. Examples of such point patterns are shown in Figure 3. Here, $\{\eta\}$ is a homogeneous Poisson process on $[0,100] \times[0,200]$ with
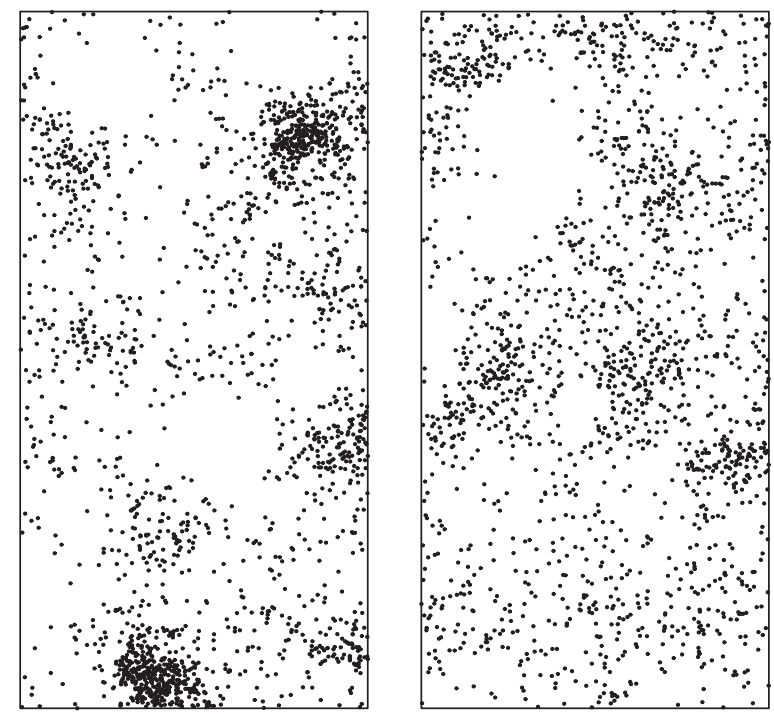

FIGURE 3: Examples of LSNCPs. Note the circular empty holes in the point patterns. For details, see the text. 
intensity $c=0.003, V(\mathrm{~d} r)=\frac{1}{3} \delta_{1}(r)+\frac{2}{3} \delta_{-1}(r)$, and the kernel is $k(\xi)=\mathbf{1}(|\xi| \leq R)$ (left plot) and $k(\xi)=\left(1-|\xi|^{3} / R^{3}\right) \mathbf{1}(|\xi| \leq R)$ (right plot), with $R=10$.

5.4.2. Log Gaussian Cox processes (LGCPS). In this subsection we consider LLCPs with a driving field of the form

$$
\Lambda(\xi)=\exp \left(\int_{\mathcal{R}} k(\xi, \eta) L(\mathrm{~d} \eta)\right),
$$

where $L$ is a Gaussian Lévy basis.

Clearly, the resulting process is an LGCP [9], [33]. If $k$ and $L$ are homogeneous, the process is stationary. In this case the random intensity function $\Lambda(\xi)$ is well defined for all $\xi \in \mathbb{R}^{d}$ and almost surely integrable if

$$
k(\xi) \leq C, \quad \xi \in \mathbb{R}^{d}, \quad \text { and } \quad \int_{\mathbb{R}^{d}} k(\xi)^{2} \mathrm{~d} \xi<\infty .
$$

The covariance function of the Gaussian field

$$
\Psi(\xi)=\int_{\mathbb{R}^{d}} k(\xi-\eta) L(\mathrm{~d} \eta)
$$

takes the form

$$
\operatorname{cov}\left(\Psi\left(\xi_{1}\right), \Psi\left(\xi_{2}\right)\right)=\int_{\mathbb{R}^{d}} k\left(\xi_{1}-\xi_{2}+\eta\right) k(\eta) \mathrm{d} \eta=c\left(\xi_{1}-\xi_{2}\right),
$$

say. Note that, under (28), $c$ is integrable. Under the mild additional assumption that the set of discontinuity points of $k$ has Lebesgue measure $0, c$ is also continuous. In Proposition 6, below, we show that any stationary LGCP with a continuous and integrable covariance function can indeed be obtained as a kernel smoothing (27) of a Gaussian Lévy basis. The proposition is a generalization of a result mentioned in [21].

Proposition 6. Any stationary Gaussian random field with continuous and integrable covariance function can be generated by a kernel smoothing of a homogeneous Lévy basis.

Proof. Let $\left\{\Psi(\xi): \xi \in \mathbb{R}^{d}\right\}$ be an arbitrary stationary zero mean Gaussian field. Let $c\left(\xi_{1}, \xi_{2}\right)=c\left(\xi_{1}-\xi_{2}\right)$ denote its covariance function, which is a function of $\xi=\xi_{1}-\xi_{2}$ due to the stationarity. Since $c$ is continuous and positive definite, it follows from Bochner's theorem that

$$
c(\xi)=\int_{\mathbb{R}^{d}} \mathrm{e}^{\mathrm{i} \xi \eta} \tau(\mathrm{d} \eta)
$$

for some nonnegative measure $\tau$. Since $c$ is integrable and symmetric, $\tau$ has a symmetric density $f$, which can be found using the inverse Fourier transform. Here $\sqrt{f}$ is continuous and a member of $L^{2}\left(\mathbb{R}^{d}\right)$. Note that, for a symmetric function defined on $\mathbb{R}^{d}$, the Fourier transform and its inverse are the same up to multiplication/division with the constant $(2 \pi)^{d / 2}$.

By the convolution theorem for the Fourier(-Plancerel) transform, we obtain

$$
\overline{(\overline{\sqrt{f}} * \overline{\sqrt{f}})}^{-1}=\overline{\overline{\sqrt{f}}}^{-1} \overline{\overline{\sqrt{f}}}^{-1}=f
$$

thus,

$$
\overline{\sqrt{f}} * \overline{\sqrt{f}}(\xi)=c(\xi) .
$$

Set $k=\overline{\sqrt{f}}$, and let $L$ denote a homogeneous Lévy basis with characteristic triplet $(0,1,0)$. Then, since the covariance function for $\int k \mathrm{~d} L$ is equal to $k * k$, our proof is complete. 
In [33, Theorem 3], conditions for ergodicity are given in the special case of a stationary LGCP. Note that, under (28), $c(\xi) \rightarrow 0$ for $\|\xi\| \rightarrow \infty$ and the conditions for ergodicity stated in [33, Theorem 3b] are satisfied.

\section{Combinations of LCPs and LLCPs}

The driving field of an LLCP has the form

$$
\Lambda(\xi)=\exp \left(\int_{\mathcal{R}} k(\xi, \eta) L_{\mathrm{J}}(\mathrm{d} \eta)\right) \exp \left(\int_{\mathcal{R}} k(\xi, \eta) L_{\mathrm{G}}(\mathrm{d} \eta)\right)=\Lambda_{\mathrm{J}}(\xi) \Lambda_{\mathrm{G}}(\xi) .
$$

It seems natural to extend the model such that the kernels used in the jump part and the Gaussian part do not need to be the same. We thereby arrive at Cox processes with a driving field of the form

$$
\Lambda(\xi)=\exp \left(\int_{\mathcal{R}} k(\xi, \eta) L_{\mathrm{J}}(\mathrm{d} \eta)\right) \Lambda_{\mathrm{G}}(\xi)
$$

where $\Lambda_{\mathrm{G}}$ is an arbitrary log Gaussian random field.

If $L_{\mathrm{J}}$ satisfies the regularity conditions of Theorem 1, we obtain

$$
\Lambda(\xi)=\exp \left(d(\xi)+\sum_{(r, \eta) \in \Phi} r k(\xi, \eta)+Y(\xi)\right)
$$

where $d(\xi)$ is a deterministic function, $\Phi$ is the atoms of a Poisson measure with intensity measure $U$, and $Y$ is an independent Gaussian field.

A related model can be found in [37] for modeling the positions of offspring in a long-leaf pine forest given the positions of the parents and information about the topography. The model in [37] is formulated conditional on the positions $\eta$ of the parents.

There are, of course, other possibilities for combining shot noise components and log Gaussian components in the driving field than the one suggested above. For instance, if $L_{\mathrm{J}}$ is a nonnegative Lévy jump basis, we may consider Cox processes driven by

$$
\begin{aligned}
\Lambda(\xi) & =\left(\int_{\mathcal{R}} k(\xi, \eta) L_{\mathrm{J}}(\mathrm{d} \eta)\right) \Lambda_{\mathrm{G}}(\xi) \\
& =\left(\int_{\mathcal{R}} k(\xi, \eta) a_{0}(\mathrm{~d} \eta)+\sum_{(r, \eta) \in N} r k(\xi, \eta)\right) \Lambda_{\mathrm{G}}(\xi) ;
\end{aligned}
$$

cf. [45]. In [13, pp. 92-100], a Cox process model of the type described in (29) has been considered, but with the Gaussian field replaced by a Boolean field. Such a model will be able to produce shot noise point patterns with empty holes generated by the Boolean field.

\section{Inhomogeneous LCPs and LLCPs}

Møller and Waagepetersen [32] suggested introducing inhomogeneity into a Cox process so that the resulting process becomes second-order intensity reweighted stationary; see [1] for details. In this section we describe four types of inhomogeneity. Only type 3 leads to second-order intensity reweighted stationary processes.

We concentrate on SNCPs with $a_{0} \equiv 0$; cf. Section 4.4.1. The interpretation of the type of inhomogeneity introduced may be facilitated by using the cluster representation of an SNCP $X$. It is not necessary for the process of cluster centers (mothers) to be locally finite in order to use this interpretation. 
Example 4. (Type 1.) The kernel is assumed to be homogeneous, $k(\xi, \eta)=k(\xi-\eta)$, while the Lévy basis satisfies $V(\mathrm{~d} r, \eta)=V(\mathrm{~d} r)$ and $v(\mathrm{~d} \eta)=c f(\eta) \mathrm{d} \eta$. If the function $f$ is nonconstant, mothers will be unevenly distributed (according to $v$ ), but the distribution of the clusters will not depend on the location in the sense that the distribution of $X_{(r, \eta)}-\eta$ does not depend on $\eta$.

Example 5. (Type 2.) The kernel is assumed to be homogeneous, $k(\xi, \eta)=k(\xi-\eta)$, while the Lévy basis satisfies $V(\mathrm{~d} r, \eta)=V(\mathrm{~d}(r / f(\eta)))$ and $\nu(\mathrm{d} \eta)=c \mathrm{~d} \eta$. In this case the mothers will be evenly distributed while the distribution of the clusters may be location dependent. A model with $(k, V)$ replaced by $k(\xi, \eta)=k(\xi-\eta) f(\eta)$ and $V(\mathrm{~d} r, \eta)=V(\mathrm{~d} r)$ will result in the same type of LCP.

Example 6. (Type 3.) The kernel is inhomogeneous of the form $k(\xi, \eta)=k(\xi-\eta) f(\xi)$, while the Lévy basis is homogeneous, $V(\mathrm{~d} r, \eta)=V(\mathrm{~d} r)$ and $\nu(\mathrm{d} \eta)=c \mathrm{~d} \eta$. The resulting LCP will be a location dependent thinning of a stationary LCP. This option has been discussed in [32] and [44] with the following log-linear specification of the function $f$ :

$$
f(\xi)=\exp (z(\xi) \beta)
$$

Here, $z(\xi)$ is a list of explanatory variables and $\beta$ is a parameter vector. Note that type 2 and type 3 inhomogeneities will typically have a similar appearance. The reason is that they can be regarded as only differing in the specification of the kernel as either of the form

$$
k(\xi, \eta)=k(\xi-\eta) f(\eta) \quad(\text { type } 2)
$$

or

$$
k(\xi, \eta)=k(\xi-\eta) f(\xi) \quad(\text { type } 3),
$$

and $k(\xi-\eta)(f(\eta)-f(\xi))$ is only nonnegligible if $\xi$ and $\eta$ are close enough so that $k(\xi-\eta)$ is nonnegligible and at the same time there is an abrupt change in $f$ between $\xi$ and $\eta$.

Example 7. (Type 4.) Inhomogeneity may also be introduced into the process by a local scaling mechanism [17], [18]. Here, the kernel is inhomogeneous,

$$
k(\xi, \eta)=k\left(\frac{\xi-\eta}{f(\eta)}\right) \frac{1}{f(\eta)^{d}},
$$

while

$$
V(\mathrm{~d} r, \eta)=V(\mathrm{~d} r) \quad \text { and } \quad v(\mathrm{~d} \eta)=c \frac{\mathrm{d} \eta}{f(\eta)^{d}} .
$$

The inhomogeneity of the resulting point process can be explained by local scaling.

In Figure 4 examples of inhomogeneous LCPs of types 1, 2, and 4 are given on $S=$ $\mathcal{R}=[0,100] \times[0,200]$. Here, $k$ is the Gaussian kernel, $(11)$, with $\sigma=2, c=\frac{1}{200}$, and $V$ is concentrated in $r=18$. The inhomogeneity function $f$ is linear in all three cases, $f(x, y)=y / 100$.

Inhomogeneity may be introduced into an LSNCP by changing $L$ or $k$ as indicated in the examples above. Compared to LCPs, the effects are now multiplicative. 

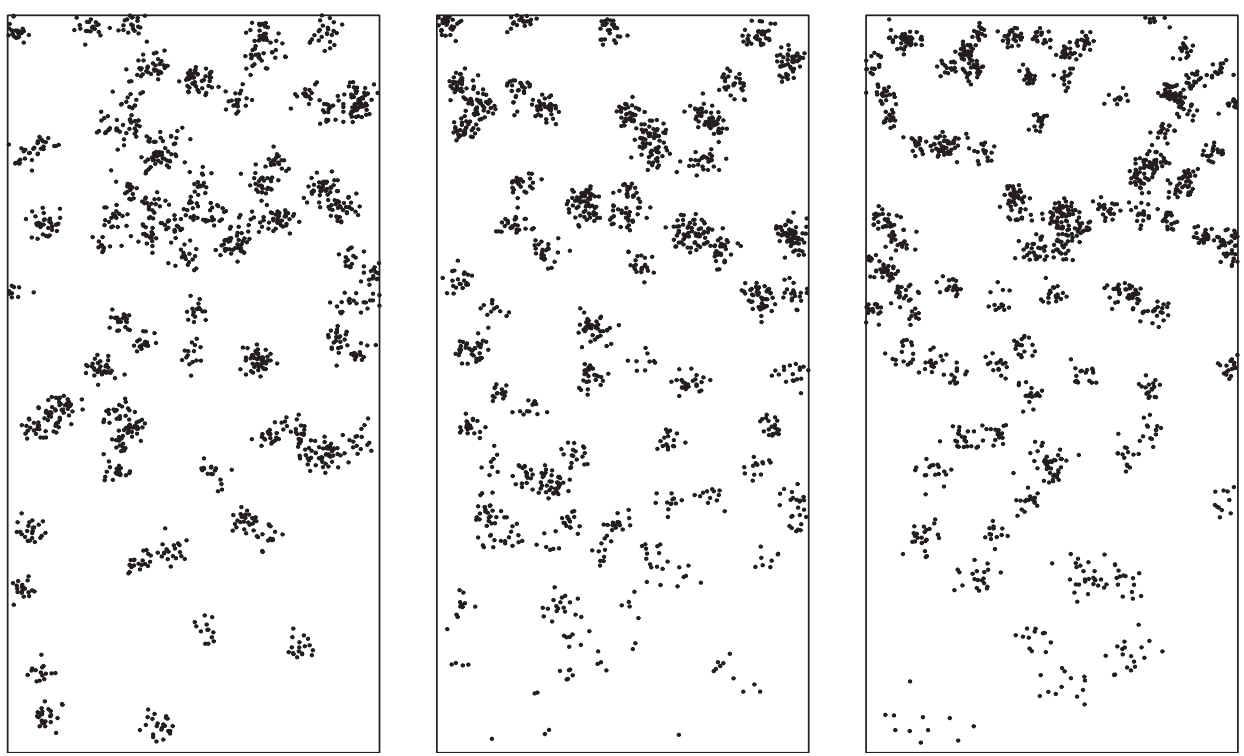

FIGURE 4: Examples of realizations of inhomogeneous LCPs: type 1 (left), type 2 (middle), and type 4 $(r i g h t)$. For details, see the text.

\section{Discussion}

During the last years, there has been some debate concerning which one of the two model classes (SNCP or LGCP) are most appropriate [27], [30], [38], [49]. The modeling framework described in the present paper provides the possibility for using models involving both SNCP and LGCP components and subsequently testing whether it is appropriate to reduce the model to a pure SNCP model or a pure LGCP model. Figure 5 summarizes the most important model classes treated in the present paper. Below, we discuss a few additional issues.

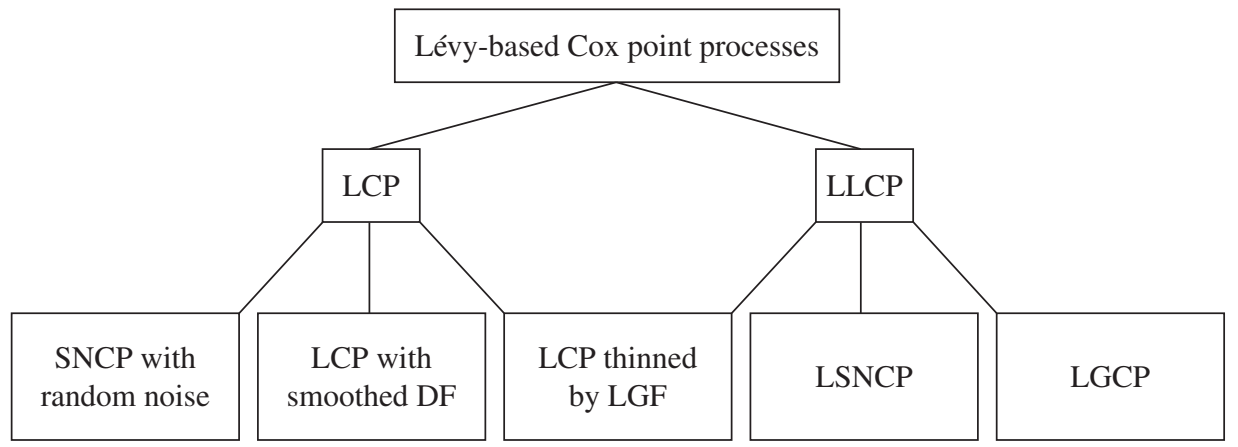

FIGURE 5: Overview of Lévy-based Cox point processes. The abbreviations DF and LGF stand for a discrete random field and a log Gaussian random field, respectively. For further details, see the text. 


\subsection{Probability densities of LCPs and LLCPs}

It is possible to derive an expression for the density of an LCP or an LLCP, using the methodology of Lévy bases. For instance, in the case of an LCP with $a_{0} \equiv 0$, the density of $X_{B}$ for $B \in B_{b}(S)$ can be written as an expansion involving complete Bell polynomials evaluated at certain cumulants. The derivation of this result utilizes (14). Unfortunately, the expansion seems to be too complicated to be of practical use for inference. The same type of conclusion has been reached for likelihood inference in $G$-SNCPs and in LGCPs; see [5, Section 4.2.1] and [33]. Closed-form expressions for the densities of other types of Cox processes are available; see [26].

\subsection{Spatio-temporal extensions}

The LCPs and LLCPs extend easily to spatio-temporal Cox processes. The set $S$ on which the process is defined is now a Borel subset of $\mathbb{R}^{d} \times \mathbb{R}$, where the last copy of $\mathbb{R}$ indicates time. The dependency on the past at time $t$ and position $x$ may be modeled using an ambit set $A_{t}(x), x \in \mathbb{R}^{d}$ and $t \in \mathbb{R}$, satisfying

$$
(x, t) \in A_{t}(x), \quad A_{t}(x) \subseteq \mathbb{R}^{d} \times(-\infty, t] .
$$

A spatio-temporal LCP is then defined by a driving intensity of the form

$$
\Lambda(x, t)=\int_{A_{t}(x)} k((x, t),(y, s)) L(\mathrm{~d}(y, s)),
$$

where $L$ is a nonnegative Lévy basis on $\mathcal{R} \subseteq \mathbb{R}^{d} \times \mathbb{R}$ and $k$ is a nonnegative weight function. Likewise, a spatio-temporal LLCP has a driving field of the form

$$
\Lambda(x, t)=\exp \left(\int_{A_{t}(x)} k((x, t),(y, s)) L(\mathrm{~d}(y, s))\right),
$$

where $L$ and $k$ no longer need to be nonnegative. Using the tools of Lévy theory, it is possible to derive moment relations as shown in the present paper for the purely spatial case [35]. This approach to spatio-temporal modeling is expected to be very flexible and has been used with success in growth modeling [23] (see also [22]). It will be interesting to investigate how it performs compared to the earlier methods described in [6], [7], [8], and [14].

\subsection{Statistical inference}

Statistical inference for Cox processes has been studied in a number of papers, including [4], [19], [27], [30], [32], and [43]. It remains to investigate to what degree known procedures, based on summary statistics, likelihood, or Bayesian reasoning, can be adjusted to deal with LCPs and LLCPs. For a stationary LCP with $\Lambda=\rho_{1}+\Lambda_{2}$, it is easy to determine the summary statistics $F, G$, and $J$ in terms of the corresponding characteristics $F_{2}, G_{2}$, and $J_{2}$ of the shot noise component with intensity field $\Lambda_{2}$. Thus,

$$
\begin{gathered}
1-F(r)=\exp \left(-\rho_{1}|B(0, r)|\right)\left(1-F_{2}(r)\right), \\
1-G(r)=\exp \left(-\rho_{1}|B(0, r)|\right)\left(\frac{\rho_{1}}{\rho_{1}+\rho_{2}}\left(1-F_{2}(r)\right)+\frac{\rho_{2}}{\rho_{1}+\rho_{2}}\left(1-G_{2}(r)\right)\right), \\
J(r)=\frac{\rho_{1}}{\rho_{1}+\rho_{2}}+\frac{\rho_{2}}{\rho_{1}+\rho_{2}} J_{2}(r) .
\end{gathered}
$$


However, in general, simple expressions for $G_{2}$ and $J_{2}$ in terms of model parameters are not available. Likewise, it does not seem to be possible to derive general closed-form expressions for $F, G$, and $J$ in the case of an LLCP.

In order to evaluate whether both a jump part and a Gaussian part is needed in an LLCP, we may consider a third-order summary statistic, suggested in [33] (for stationary point processes),

$$
z(t)=\frac{1}{\pi^{2} t^{4}} \int_{\|\xi\| \leq t} \int_{\|\zeta\| \leq t} \frac{\rho^{(3)}(\xi, \zeta)}{\left(\rho^{(1)}\right)^{3} g(\xi) g(\zeta) g(\xi-\zeta)} \mathrm{d} \xi \mathrm{d} \zeta, \quad t>0
$$

where the following abbreviated notation is used, due to the stationarity:

$$
\begin{gathered}
g\left(\xi_{1}, \xi_{2}\right)=g\left(\xi_{2}-\xi_{1}\right), \\
\rho^{(3)}\left(\xi_{1}, \xi_{2}, \xi_{3}\right)=\rho^{(3)}\left(\xi_{2}-\xi_{1}, \xi_{3}-\xi_{1}\right) .
\end{gathered}
$$

When computing the integrand in $z(t)$ for an LLCP, we obtain

$$
\begin{aligned}
& \frac{\rho^{(3)}\left(\xi_{1}, \xi_{2}, \xi_{3}\right)}{\left(\rho^{(1)}\right)^{3} g\left(\xi_{1}, \xi_{2}\right) g\left(\xi_{2}, \xi_{3}\right) g\left(\xi_{1}, \xi_{3}\right)} \\
& =\frac{\mathrm{E}\left(\prod_{i=1}^{3} \Lambda_{\mathbf{J}}\left(\xi_{i}\right)\right) \prod_{i=1}^{3} \mathrm{E}\left(\Lambda_{\mathbf{J}}\left(\xi_{i}\right)\right)}{\mathrm{E}\left(\Lambda_{\mathbf{J}}\left(\xi_{1}\right) \Lambda_{\mathbf{J}}\left(\xi_{2}\right)\right) \mathrm{E}\left(\Lambda_{\mathbf{J}}\left(\xi_{2}\right) \Lambda_{\mathrm{J}}\left(\xi_{3}\right)\right) \mathrm{E}\left(\Lambda_{\mathrm{J}}\left(\xi_{1}\right) \Lambda_{\mathrm{J}}\left(\xi_{3}\right)\right)},
\end{aligned}
$$

where $\Lambda_{\mathrm{J}}(\xi)=\exp \left(\int_{\mathcal{R}} k(\xi, \eta) L_{\mathrm{J}}(\mathrm{d} \eta)\right)$ is the part of the driving intensity originating from the pure-jump part of the Lévy basis. Thus, this characteristic of $X$ is not influenced by the Gaussian part of the model. In particular, $z \equiv 1$ for LGCPs. A nonparametric unbiased estimator of $z(t)$ has been derived in [33, Theorem 2].

An assessment of the full potential of the new modeling framework described in the present paper will also require more detailed studies of inhomogeneity and practical experience with concrete applications of the models.

\section{Acknowledgements}

We are grateful to Ole E. Barndorff-Nielsen for sharing his thoughts with us. This research has been supported by grants from the Carlsberg Foundation and the Danish Natural Science Research Council.

\section{References}

[1] Baddeley, A. J., Møller, J. and Waagepetersen, R. (2000). Non- and semi-parametric estimation of interaction in inhomogeneous point patterns. Statist. Neerlandica 54, 329-350.

[2] BarndorfF-Nielsen, O. E. ANd Schmiegel, J. (2004). Lévy based tempo-spatial modeling; with applications to turbulence. Uspekhi Mat. Nauk 159, 63-90.

[3] Barndorff-Nielsen, O. E., Blesild, P. And Schmiegel, J. (2004). A parsimonious and universal description of turbulent velocity increments. Europ. Phys. J. B 41, 345-363.

[4] Best, N. G., Ickstadt, K. And Wolpert, R. L. (2000). Spatial Poisson regression for health and exposure data measured at disparate resolutions. J. Amer. Statist. Assoc. 95, 1076-1088.

[5] BRIX, A. (1999). Generalized gamma measures and shot-noise Cox processes. Adv. Appl. Prob. 31, 929-953.

[6] Brix, A. ANd Chadeuf, J. (2002). Spatio-temporal modeling of weeds by shot-noise G Cox processes. Biom. J. 44, 83-99.

[7] Brix, A. And Diggle, P. J. (2001). Spatio-temporal prediction for log-Gaussian Cox processes. J. R. Statist. Soc. B 63, 823-841.

[8] Brix, A. AND Møller, J. (2001). Space-time multitype log Gaussian Cox processes with a view to modeling weed data. Scand. J. Statist. 28, 471-488. 
[9] Coles, P. And Jones, B. (1991). A lognormal model for the cosmological mass distribution. Monthly Notes R. Astronomical Soc. 248, 1-13.

[10] Comtet, L. (1970). Analyse Combinatoire. Tomes I, II. Presses Universitaires de France, Paris.

[11] Cox, D. R. (1955). Some statistical models related with series of events. J. R. Statist. Soc. B 17, 129-164.

[12] Daley, D. J. And Vere-Jones, D. (1988). An Introduction to the Theory of Point Processes. Springer, New York.

[13] Diggle, P. J. (2003). Statistical Analysis of Spatial Point Patterns, 2nd edn. Hodder Arnold, London.

[14] Diggle, P., Rowlingson, B. AND Su, T. (2005). Point process methodology for on-line spatio-temporal disease surveillance. Environmetrics 16, 423-434.

[15] Federer, H. (1969). Geometric Measure Theory. Springer, Berlin.

[16] Ferguson, T. S. and Klass, M. J. (1972). A representation of independent increment processes without Gaussian components. Ann. Math. Statist. 43, 1634-43.

[17] Hahn, U. (2007). Global and Local Scaling in the Statistics of Spatial Point Processes. Habilitationsschrift, Institut für Mathematik, Universität Augsburg.

[18] Hahn, U., Jensen, E. B. V., van Lieshout, M. N. M. and Nielsen, L. S. (2003). Inhomogeneous spatial point processes by location dependent scaling. Adv. Appl. Prob. 35, 319-336.

[19] Heikkinen, J. And Arjas, E. (1998). Non-parametric Bayesian estimation of a spatial Poisson intensity. Scand. J. Statist. 25, 435-450.

[20] Hellmund, G. (2005). Lévy driven Cox processes with a view towards modelling tropical rain forest. Masters Thesis, Department of Mathematical Sciences, University of Aarhus.

[21] Higdon, D. (2002). Space and space-time modeling using process convolutions. In Quantitative Methods for Current Environmental Issues, eds C. W. Anderson et al., Springer, London, pp. 37-56.

[22] Jensen, E. B. V., Jónsdóttir, K. Y., Schmiegel, J. And Barndorff-Nielsen, O. E. (2007). Spatio-temporal modeling - with a view to biological growth. In Statistics of Spatio-Temporal Systems, eds B. F. Finkenstadt et al., Chapman \& Hall/CRC, Boca Raton, FL, pp. 47-75.

[23] Jónsdóttir, K. Y., Schmiegel, J. And Jensen, E. B. V. (2008). Lévy-based growth models. Bernoulli 14, 62-90.

[24] Khoshnevisan, D. (2002). Multiparameter Processes. An Introduction to Random Fields. Springer, New York.

[25] Matérn, B. (1986). Spatial Variation. (Lecture Notes Statist. 36), 2nd edn. Springer, Berlin.

[26] McCullagh, P. and Møller, J. (2006). The permanental process. Adv. Appl. Prob. 38, 873-888.

[27] MøLler, J. (2003). A comparison of spatial point process models in epidemiological applications. In Highly Structured Stochastic Systems, eds P. J. Green et al., Oxford University Press, pp. 264-268.

[28] Møller, J. (2003). Shot noise Cox processes. Adv. Appl. Prob. 35, 614-640.

[29] Møller, J. And Torrisi, G. L. (2005). Generalised shot noise Cox processes. Adv. Appl. Prob. 37, 48-74.

[30] Møller, J. and Waagepetersen, R. P. (2002). Statistical inference for Cox processes. In Spatial Cluster Modelling, eds A. B. Lawson and D. Denison, Chapman \& Hall/CRC, Boca Raton, FL, pp. 37-60.

[31] Møller, J. and WaAgepetersen, R. P. (2003). Statistical Inference and Simulation for Spatial Point Processes. Chapman \& Hall/CRC, Boca Raton, FL.

[32] Møller, J. and Waagepetersen, R. P. (2007). Modern statistics for spatial point processes. Scand. J. Statist. 34, 643-684.

[33] Møller, J., Syversveen, A. R. and Waagepetersen, R. P. (1998). Log Gaussian Cox processes. Scand. J. Statist. 25, 451-482.

[34] Neyman, J. And Scott, E. L. (1958). Statistical approach to problems of cosmology. J. R. Statist. Soc. B 20, $1-43$.

[35] Prokešová, M., Hellmund, G. And Jensen, E. B. V. (2006). On spatio-temporal Lévy based Cox processes. In Proc. $S^{4} G$, Internat. Conf. Stereology, Spatial Statist. Stoch. Geometry (Prague, June 2006), eds R. Lechnerová, et al., Union Czech Mathematicians and Physicists, Prague, pp. 111-116.

[36] Rajput, R. S. And Rosinski, J. (1989). Spectral representation of infinitely divisible processes. Prob. Theory Relat. Fields 82, 451-487.

[37] Rathburn, S. R. And Cressie, N. (1994). A space-time survival point process for longleaf pine forest in southern Georgia. J. Amer. Statist. Assoc. 89, 1164-1174.

[38] RichardSON, S. (2003). Spatial models in epidemiological applications. In Highly Structured Stochastic Systems, eds P. J. Green, et al., Oxford University Press, pp. 237-259.

[39] Rosinski, J. (2008). Simulation of Lévy processes. In Encyclopedia of Statistics in Quality and Reliability: Computational Intensive Methods and Simulation. John Wiley.

[40] Sato, K. -I. (1999). Lévy Processes and Infinitely Divisible Distributions. Cambridge University Press.

[41] Stoyan, D., Kendall, W. S. And Mecke, J. (1995). Stochastic Geometry and Its Applications, 2nd edn. John Wiley, Chichester.

[42] Thomas, M. (1949). A generalization of Poisson's binomial limit for use in ecology. Biometrika 36, 18-25.

[43] Van Lieshout, M. N. M. ANd Baddeley, A. J. (1996). A nonparametric measure of spatial interaction in point patterns. Statist. Neerlandica 50, 344-361. 
[44] Waagepetersen, R. P. (2005). Discussion of the paper by Baddeley, Turner, Møller and Hazelton (2005). J. R. Statist. Soc. B 67, 662.

[45] WaAgepetersen, R. P. (2007). Personal communication.

[46] Walker, S. and Damien, P. (2000). Representations of Lévy processes without Gaussian components. Biometrika 87, 477-483.

[47] Wiegand, T., Gunatilleke, S., Gunatilleke, N. and OKuda, T. (2007). Analyzing the spatial structure of a Sri Lankan tree species with multiple scales of clustering. Ecology 88, 3088-3102.

[48] Wolpert, R. L. (2001). Lévy moving averages and spatial statistics. Lecture. Slides available at http:// citeseer.ist.psu.edu/wolpert01leacutevy.html.

[49] WolPERT, R. L. AND ICKSTADT, K. (1998). Poisson/gamma random fields models for spatial statistics. Biometrika 85, 251-267.

[50] Wolpert, R. L. AND IcKstadt, K. (1998). Poisson/gamma random fields models for spatial statistics. Simulation of Lévy random fields. In Practical Nonparametric and Semiparametric Bayesian Statistics, (Lecture Notes Statist. 133), eds D. Dey et al., Springer, New York, pp. 227-242. 